AUS DEM LEHRSTUHL

FÜR INNERE MEDIZIN I

Prof. Dr. Martina Müller-Schilling

DER FAKULTÄT FÜR MEDIZIN

DER UNIVERSITÄT REGENSBURG

\title{
DYSBALANCE IN SYMPATHETIC NEUROTRANSMITTER RELEASE AND \\ ACTION IN CIRRHOTIC RATS: IMPACT OF EXOGENOUS NEUROPEPTIDE Y
}

\author{
Inaugural - Dissertation \\ zur Erlangung des Doktorgrades \\ der Medizin \\ der \\ Fakultät für Medizin \\ der Universität Regensburg
}

vorgelegt von

Peter Dietrich 
AUS DEM LEHRSTUHL

FÜR INNERE MEDIZIN I

Prof. Dr. Martina Müller-Schilling

DER FAKULTÄT FÜR MEDIZIN

DER UNIVERSITÄT REGENSBURG

DYSBALANCE IN SYMPATHETIC NEUROTRANSMITTER RELEASE AND ACTION IN CIRRHOTIC RATS: IMPACT OF EXOGENOUS NEUROPEPTIDE Y

\author{
Inaugural - Dissertation \\ zur Erlangung des Doktorgrades \\ der Medizin \\ der \\ Fakultät für Medizin \\ der Universität Regensburg
}

vorgelegt von

Peter Dietrich

2013 
Dekan:

1. Berichterstatter:

2. Berichterstatter:

Tag der mündlichen Prüfung:
Prof. Dr. Dr. Torsten E. Reichert

Prof. Dr. Reiner Wiest

Prof. Dr. Anja Bosserhoff

15.10. und 17.10.2013 


\section{Inhaltsverzeichnis}

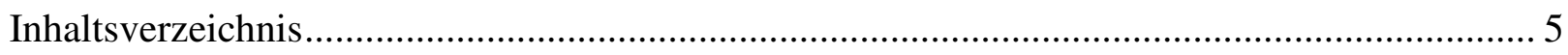

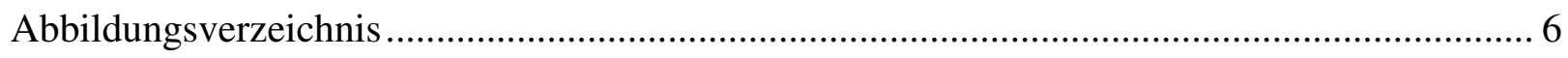

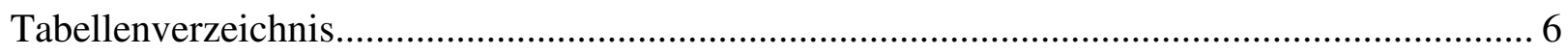

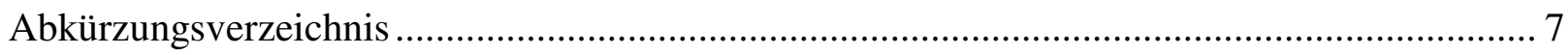

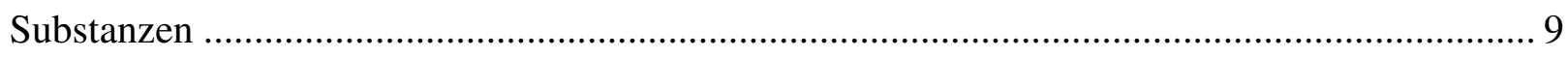

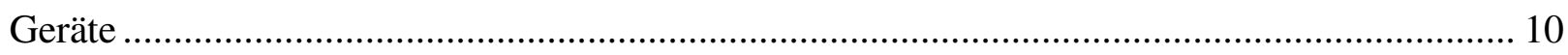

Zusammenfassung der Arbeit in deutscher Sprache

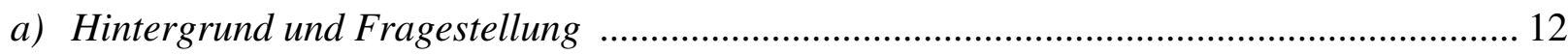

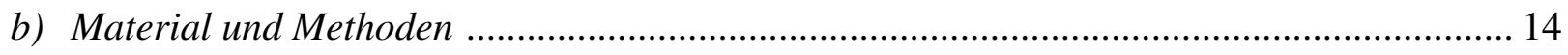

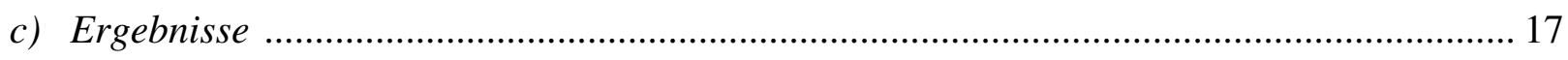

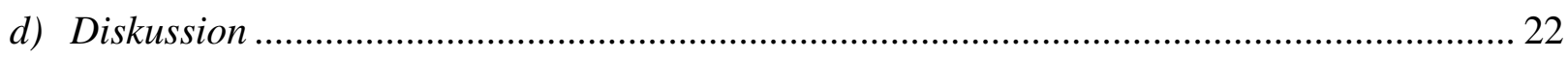

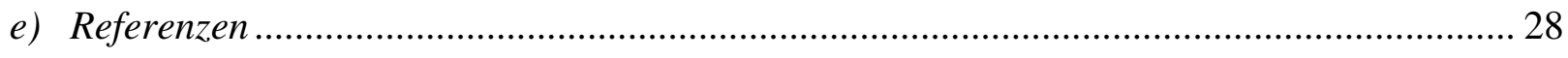

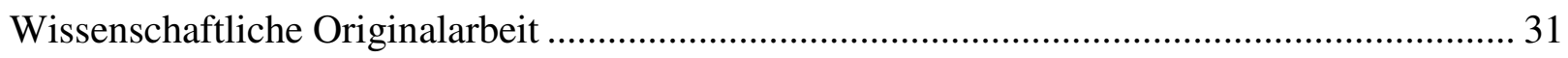

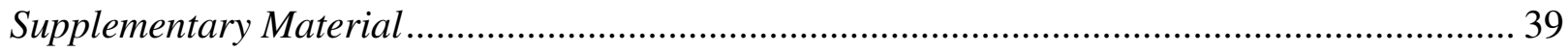

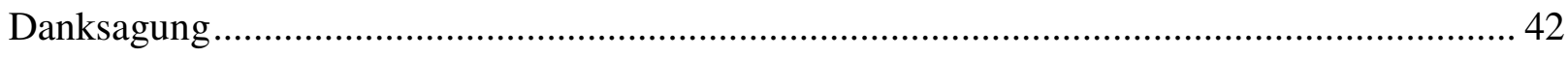




\section{Abbildungsverzeichnis}

Abbildung 1: Vorrichtung zur periarteriellen Nervenstimulation

Abbildung 2: Vaskuläre Druckantworten und

Neurotransmitter-Freisetzung im 1. Perfusionszyklus 18

Abbildung 3: $\quad$ Druckantwort, NA-Sekretion und der Einfluss von exogenem NPY im 2. Perfusionszyklus.

Abbildung 4: „Erholung“ der PNS-induzierten Druckantwort mit und ohne exogenem NPY

Abbildung 5: Hypothese der dysregulierten sympathischen Neurotransmitter-Sekretion im mesenterialen arteriellen Gefäßbett bei portaler Hypertension.

\section{Tabellenverzeichnis}

Tabelle 1: $\quad$ Basalwerte und hämodynamische Parameter

im 1. Perfusionszyklus 


\section{Abkürzungsverzeichnis}

\begin{tabular}{|c|c|}
\hline AMS & Arteria mesenterica superior \\
\hline $\mathrm{ACH}$ & Acetylcholin \\
\hline AT1-R & Angiotensin-II(Typ1)-Rezeptor \\
\hline ATP & Adenosintriphosphat \\
\hline cAMP & Cyclisches Adenosinmonophosphat \\
\hline DPP-IV & Dipeptidyl-Peptidase-IV \\
\hline ET-1 & Endothelin-1 \\
\hline ET-3 & Endothelin-3 \\
\hline eNOS & Epitheliale NO-Synthase \\
\hline HSP-90 & Hitze-Schock-Protein-90 \\
\hline HZS & Hyperdynames Zirkulationssyndrom \\
\hline IP3 & Inositol-1,4,5-triphosphat \\
\hline KG & Körpergewicht \\
\hline $\mathrm{LC}$ & Leberzirrhose \\
\hline LPS & Lipopolysaccharid \\
\hline MLC & Myosin-Leichte-Kette \\
\hline MLCK & Myosin-Leichte-Kette-Kinase \\
\hline MLCP & Myosin-Leichte-Kette-Phosphatase \\
\hline NA & Noradrenalin \\
\hline nNOS & Neuronale NO-Synthase \\
\hline $\mathrm{NO}$ & Stickstoffmonoxid \\
\hline NOS & NO-Synthase \\
\hline NPY & Neuropeptid Y \\
\hline n.s. & Nicht signifikant \\
\hline $\mathrm{PC}$ & Perfusionszyklus \\
\hline PKA & Proteinkinase $\mathrm{A}$ \\
\hline
\end{tabular}




\begin{tabular}{|l|l|}
\hline PKC & Proteinkinase C \\
\hline PKG & Proteinkinase G \\
\hline PLA $_{2}$ & Phospholipase $\mathrm{A}_{2}$ \\
\hline PLC & Phospholipase C \\
\hline PNS & Periarterielle Nervenstimulation \\
\hline PR & Pressure-response=Druckantwort \\
\hline PVL & Portal-Venen-Ligatur \\
\hline r & Korrelationskoeffizient (Pearson) \\
\hline R & Determinationskoeffizient \\
\hline RAAS & Renin-Angiotension-Aldosteron-System \\
\hline SEM & Standardabweichung \\
\hline Sham & Kontrolltier \\
\hline SNS & Sympathisches Nervensystem \\
\hline
\end{tabular}




\section{Substanzen}

\begin{tabular}{|l|l|}
\hline Aqua Resist & VWR-International \\
\hline Calciumchlorid Dihydrat & J.T. Baker, Phillipsburg, USA \\
\hline Carbogen & Linde Technische Gase \\
\hline D(+)-Glucose Monohydrat & J.T. Baker, Phillipsburg, USA \\
\hline Dimethyl-Sulfoxid (DMSO) & Fluka, Steinheim \\
\hline Domitor (0,2 ml=0,05 mg Medetomidin) & Pfizer, Karlsruhe \\
\hline EDTA & Sigma, Steinheim \\
\hline Ethanol (67\%) & J.T. Baker, Phillipsburg, USA \\
\hline Fentanyl & Curamed Pharma \\
\hline Kaliumchlorid & Merck, Darmstadt \\
\hline Kaliumhydrogenphosphat & J.T. Baker, Phillipsburg, USA \\
\hline Magnesiumsulfat-7-Hydrat & J.T. Baker, Phillipsburg, USA \\
\hline Midazolam & Curamed Pharma \\
\hline NaCl, 0,9\%, steril & Braun, Melsungen \\
\hline Natriumchlorid, Pulver & J.T. Baker, Phillipsburg, USA \\
\hline Natriumhydrogencarbonat & J.T. Baker, Phillipsburg, USA \\
\hline Neuropeptid Y & Calbiochem (Merck), Darmstadt \\
\hline Phenobarbital & Sigma-Aldrich Chemie GmbH \\
\hline
\end{tabular}




\section{Geräte}

\begin{tabular}{|c|c|}
\hline Autoklav & Wolf, Geislingen \\
\hline Carbogen - Flasche & Linde Technische Gase \\
\hline Drei-Wege Hahn aus Kunststoff & NeoLab, Heidelberg \\
\hline Einhängethermostat C10 & Haake Deutschland \\
\hline Einmalspritzen, Discardit 2/5/10 ml steril & Becton-Dickinson, San Jose, USA \\
\hline Eismaschine, UBE 125 & Ziegra, Isernhagen \\
\hline Glasbehälter (div. Volumina) & Schott, Mainz \\
\hline Laborwaage, SC 2020 & Ohaus, Giessen \\
\hline Magnetrührer, IKAMag REO & Heidolph, Kehlheim \\
\hline Microlance Kanülen & Becton-Dickinson, San Jose, USA \\
\hline Nahtmaterial, vicryl, violett 3 & Ethicon, Norderstedt \\
\hline Nervenstimulations-Vorrichtung & (Eigenbau) P. Dietrich \\
\hline P-23-DbPerfussionsdruck-Messelektrode (Transducer) & Statham, Oxnard, CA, USA \\
\hline Parafilm M (250 ft x 4 in) & $\begin{array}{lll}\text { Pechiney Plastic } & \text { Packaging } & \text { Inc., } \\
\text { Neenah, USA } & & \end{array}$ \\
\hline Pasteurpipetten, 5/10/20 ml & Brand, Wertheim \\
\hline Perfusionsanlage & $\begin{array}{l}\text { (Eigenbau) Prof. Dr. med R. Wiest, } \\
\text { Universitätsspital Bern }\end{array}$ \\
\hline Perfusionsschlauch, $140 \mathrm{~cm}$ & Heidelberger Verlängerung \\
\hline Perfusionsschlauch, Mikro PE: 0,5x1,0 mm & Reichelt Chemietechnik, Heidelberg \\
\hline Pipetten $(10 / 100 / 1000 \mu \mathrm{l})$ & Eppendorf, Hamburg \\
\hline Pipettenspitzen $(10 / 100 / 1000 \mu \mathrm{l})$ & Eppendorf, Hamburg \\
\hline PowerLab 4/20 & PowerLab/ADInstruments, Heidelberg \\
\hline Präparationsbesteck & Aesculap, Tuttlingen \\
\hline Pumpe IPC 8-Kanal & Ismatec, Wertheim-Mondfeld \\
\hline Pumpenschläuche, Tygon R-3607 & Ismatec, Wertheim-Mondfeld \\
\hline Reaktionsgefäße 1 und $2 \mathrm{ml}$ & Eppendorf, Hamburg \\
\hline Reaktionsgefäße 15 und $50 \mathrm{ml}$ & Falcon, Heidelberg \\
\hline Reinstwasseranlage, Milli-Q-Plus & $\begin{array}{l}\text { Ultra Pure Water Systems, Billerica, } \\
\text { USA }\end{array}$ \\
\hline Schlauchverbindungen $2-3,5 \mathrm{~mm}$ & NeoLab, Heidelberg \\
\hline
\end{tabular}




\begin{tabular}{|l|l|}
\hline Schüttler REAX 2 & Heidolph, Schwabach \\
\hline Stickstoff & Linde Technische Gase \\
\hline Telacomp, Tupfer, x-ray, 10x10 cm & Hartmann, Heidelberg \\
\hline Transducer, BLPR one 05A & $\begin{array}{l}\text { World Precision Instruments Inc., } \\
\text { Sarasota, USA }\end{array}$ \\
\hline T-Stücke, Mikro 2,3-3,5 mm & NeoLab, Heidelberg \\
\hline
\end{tabular}


DYSBALANCE IN SYMPATHETIC NEUROTRANSMITTER RELEASE AND ACTION IN CIRRHOTIC RATS: IMPACT OF EXOGENOUS NEUROPEPTIDE Y

\section{DYSREGULATION DER SYMPATHISCHEN NEUROTRANSMITTER- FREISETZUNG UND -WIRKUNG BEI EXPERIMENTELLER LEBERZIRRHOSE: DER EINFLUSS VON EXOGENEM NEUROPEPTID Y}

\section{a. Hintergrund und Fragestellung}

Die gesteigerte splanchnische arterielle Vasodilatation bei portaler Hypertension wird auf eine vermehrte Produktion vasodilatativer Substanzen einerseits sowie eine vasokonstriktive Hyporeaktivität andererseits zurückgeführt [1]. Letzterer Mechanismus kann teilweise mit einer defekten intrazellulären vasokonstriktiven Signaltransduktion der glatten Gefäßmuskelzelle erklärt werden [2]. Das Sympathische Nervensystem (SNS) wird hierbei konsekutiv hochreguliert. Dieser vasokonstriktive Kompensationsmechanismus ist von prognostischer Signifikanz: So korrelieren erhöhte Serumkonzentrationen von Noradrenalin (NA) mit dem Überleben von Patienten mit Leberzirrhose [3, 4]. Es gibt deutliche Evidenz dafür, dass bei portaler Hypertension die systemischen NA-Plasmakonzentrationen hauptsächlich von der mesenterialen NA-Produktion abhängen [5]. Aber anders als bei systemischen NAKonzentrationen sind Daten $\mathrm{zu}$ lokaler splanchnischer Neurotransmitter-Produktion und -Sekretion sehr spärlich vorhanden und zudem kontrovers [6-12]. 
Neuropeptid Y (NPY) wird in sympathischen Nervenendigungen gespeichert und als KoTransmitter zusammen mit NA ausgeschüttet, um via G-Protein gekoppelter postsynaptischer $\mathrm{Y}_{1}$-Rezeptoren die $\alpha_{1}$-adrenerge vasokonstriktive Wirkung von NA zu potenzieren [13].

NPY vermittelt hierbei eine Sensibilisierung der glatten Gefäßmuskelzell-Antwort auf NA, auch wenn der exakte intrazelluläre Signalweg noch unbekannt ist [14]. In unserer Arbeitsgruppe wurde bereits gezeigt, dass NPY eine durch exogenes Noradrenalin (NA) induzierte $\alpha_{1}$-adrenerge mesenteriale arterielle Vasokonstriktion in zirrhotischen Ratten in signifikant höherem Maße steigert als bei gesunden Tieren $[15,16]$.

Eine längere Stimulation von glatten Gefäßmuskelzellen mit adrenergen Agonisten kann in einer herabgesetzten Antwort auf Katecholamine resultieren - dieses Phänomen wurde Desensibilisierung genannt. Somit könnten anhaltend erhöhte Plasmaspiegel sympathischer Neurotransmitter, wie man sie beispielsweise beim Phäochromozytom, aber auch bei Herzinsuffizienz und Leberzirrhose findet, zu vaskulärer Desensibilisierung führen und dadurch zu einer Aggravation der splanchnischen Vasodilatation bei Leberzirrhose beitragen $[17,18]$.

Im isolierten mesenterialen Gefäßbett der Ratte kann eine elektrische Stimulation der sympathischen Nerven eine extensive und/oder lang anhaltende Aktivität des sympathischen Nervensystems (SNS) nachahmen. Dies wurde bereits bei gesunden Ratten untersucht. Es kommt dabei zur splanchnischen arteriellen Vasokonstriktion und damit zur Induktion einer messbaren vaskulären Druckantwort, welche durch die Sekretion von NA und NPY vermittelt wird [19, 20]. Die Nervenstimulation als physiologischer Stimulus der sympathoadrenergen Modellierung des vaskulären Tonus wurde jedoch bisher nur unzureichend bei experimenteller portaler Hypertension untersucht. Insbesondere liegen wenige Daten über die direkte Messung der lokalen splanchnischen Freisetzung sympathischer Neurotransmitter und deren hämodynamischen Auswirkungen vor.

Dieses Experiment sollte daher die Frage klären, ob sich der Effekt der $\alpha_{1}$-adrenergen Desensibilisierung durch Katecholaminausschüttung unter periarterieller elektrischer Nervenstimulation bei gesunden und portal hypertensiven Tieren sowie zirrhotischen Tieren unterscheidet. Ein weiteres Ziel war die Charakterisierung der lokalen mesenterialen Sekretion der sympathischen Neurotransmitter NA und NPY. Schließlich sollte der Einfluss von exogen zugeführten NPY auf die durch periarterielle Nervenstimulation induzierte NeurotransmitterFreisetzung mit konsekutiver Druckantwort bei experimenteller portaler Hypertension untersucht werden. 


\section{b. Material und Methoden}

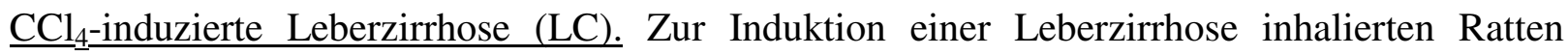
(Charles-River) dreimal wöchentlich Tetrachlorkohlenstoff $\left(\mathrm{CCl}_{4}\right)$ und erhielten begleitend mit Phenobarbital (0.35g/L) versetztes Trinkwasser. Dieses Vorgehen führt innerhalb von ca. 12 bis 16 Wochen zu einer mikronodulären Leberzirrhose.

Induktion einer portalen Hypertension durch Portal-Venen-Ligatur (PVL). Neben zirrhotischen Ratten wurde auch ein Modell mit prähepatischer portaler Hypertension verwendet, welches seit langem in unserem Labor etabliert ist $[15,16]$. Hierzu wurde bei PVL-Tieren nach einer medianen Laparotomie eine Ligatur um eine der V. portae anliegende 20-G-Kanüle vorgenommen. Durch Entfernung der Kanüle wurde eine kalibrierte und standardisierte Stenose induziert.

In-vitro Perfusion. Die Präparation des mesenterialen Gefäßbettes erfolgte nach der Methode von McGregor [21]. Nach Kanülierung der A. mesenterica superior (PE60) wurde hierbei das Mesenterium inklusive dem mesenterialen Gefäßbett entnommen, mit Parafilm umhüllt und in einer gewärmten $\left(37^{\circ} \mathrm{C}\right)$ Perfusionskammer platziert. Dort wurde das Präparat mittels oxygenierter $\left(95 \% \mathrm{O}_{2}, 5 \% \mathrm{CO}_{2}\right)$,Krebs“-Lösung $\left(37^{\circ} \mathrm{C}\right)$ mit einer konstanten Flussrate von 4 $\mathrm{ml} / \mathrm{min}$ perfundiert. Das Perfusat wurde kontinuierlich aus der Perfusionskammer abgeleitet. Die Druckmessung erfolgte mittels eines P-23-Db-Transducers und wurde PC-gestützt online unter Verwendung des Programms PowerLab aufgezeichnet. Unter den genannten Bedingungen lieferte diese Anordnung für mindestens 4 Stunden zuverlässige Daten.

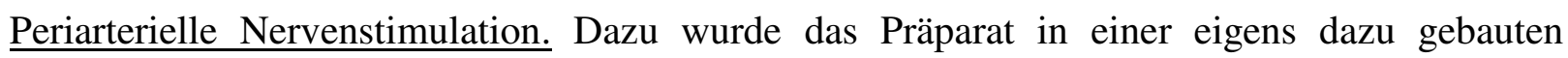
Vorrichtung aus Edelstahl fixiert (Abb. 1). Eine Platinnetzelektrode (Plus-Pol) wurde flach auf einer beheizten Grundplatte $\left(37^{\circ} \mathrm{C}\right)$ fixiert. Auf diese wurde dann das perfundierte Präparat gelegt, und eine Drahtelektrode (Minus-Pol) wurde parallel entlang der A. mesenterica superior (SMA) mit Sympathikus-Hauptnervenstranges (Plexus mesentericus superior) mit konstantem Druck (erreicht durch eine Feder) angelegt, sodass Strom fließen konnte. Die elektrische Stimulation erfolgte durch einen Nervenstimulator (I-ZQ4V, Hugo Sachs Electronics) in Pulsen von $50 \mathrm{~V}$ und $1 \mathrm{~ms}$ Breite für je 30 Sekunden bei einer Stimulationsfrequenz von $33 \mathrm{~Hz}$. Mit dieser elektrischen Nervenstimulation werden im mesenterialen Gefäßbett maximale 
Druckantworten induziert, welche nicht durch Erhöhung von Frequenz, Dauer oder Amplitude weiter gesteigert werden können. Dies konnte durch zahlreiche Vorversuche ermittelt werden.

\begin{abstract}
Abbildung 1: Vorrichtung zur periarteriellen Nervenstimulation. Die Netz-Elektrode (Pfeil A) lag auf einer beheizten Grundplatte (Pfeil B). Darauf wurde das perfundierte mesenteriale Gefäßbett (Pfeil C) gelegt. Eine fixierte Drahtelektrode (Pfeil D) wurde parallel entlang der A. mesenterica superior und des mesenterialen SympathikusHauptnervenstranges (Plexus mesentericus superior) mit konstantem Druck (erreicht durch eine Feder, Pfeil E) angelegt, sodass Strom fließen konnte.
\end{abstract}

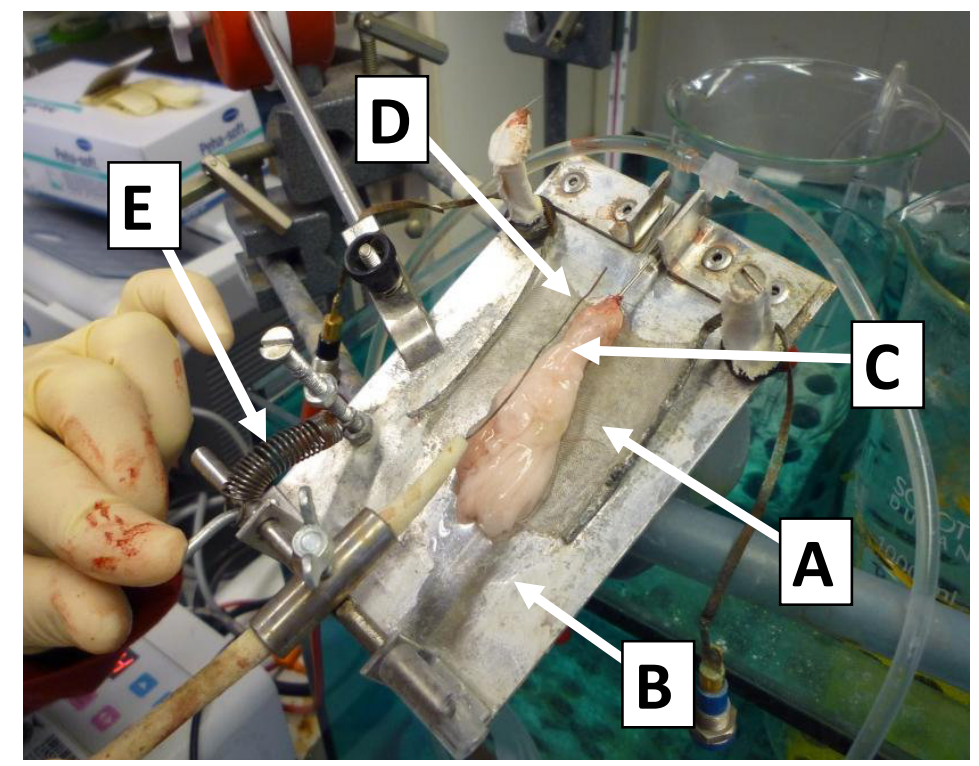

Quantifizierung von Noradrenalin (NA). Proben mit $3 \mathrm{ml}$ des Subperfusates wurden jeweils direkt gesammelt (die Sammelröhrchen enthielten $60 \mu 1$ GSH/EGTA-Lösung als Antioxidans). Zur NA-Messung verwendeten wir ein HPLC-System (Shimadzu, Duisburg, Germany). 1-1.5 ml Perfusat-Proben wurden mittels kommerziell erhältlichen Kit (ClinRep® Catecholamines in plasma, Recipe, Munich, Germany) analysiert. Die minimal messbare Konzentration lag bei 5 $\mathrm{pg} / \mathrm{ml} \mathrm{NA}$.

Quantifizierung von Neuropeptid Y (NPY). Hierzu wurde ein kommerzielles RIA-Kit von der Firma Eurodiagnostica (EURIA-NPY) verwendet. Kreuzreaktionen mit anderen Peptiden waren unter $0.1 \%$. Die minimal messbare Konzentration lag bei 3 pmol/l, die mittlere recovery range war $75-88 \%$.

Protokoll I: Vaskuläre Desensibilisierung nach repetitiver periarterieller Nervenstimulation (PNS) und Neurotransmitter-Sekretion. Mit Hilfe dieses Protokolls sollte das Ausmaß der durch repetitive PNS induzierbaren Degradation der vaskulären Druckantwort untersucht werden [Kontrollgruppe=Con: $n=19$, PVL: $n=12$, LC: $n=19]$. Nach einer Vorinkubationszeit von 20-30 Minuten bis zur Etablierung eines stabilen Baseline-Druckes bei einer Perfusionsgeschwindigkeit von $4 \mathrm{ml} / \mathrm{min}$ führten wir in einem 1. Perfusionszyklus (1. PC) 15 repetitive PNS durch. Der Abstand zwischen den einzelnen Stimulationen betrug jeweils 8 Minuten. Um die endogene Freisetzung von NA und NPY zu untersuchen, wurden jeweils nach 
der 3., 6., 9., 12. und 15. PNS in einer weiteren Versuchsreihe Perfusat-Proben wie oben beschrieben gesammelt [Con: $n=12, L C: n=12]$. Bei PVL-Tieren wurden aus folgenden Gründen keine NA-Bestimmungen durchgeführt: In PVL-Ratten entwickelt sich der Zustand des hyperdynamen Zirkulationssyndromes akut innerhalb von 3-5 Tagen [22]. Danach nimmt das Ausmaß der portalen Hypertension in diesem Modell sukzessive aufgrund der Ausbildung von Kollateralen ab. Die kompensatorische Hochregulation des SNS und damit auch die veränderte Neurotransmitter-Sekretion bei Leberzirrhose ist jedoch ein sich chronisch entwickelnder Zustand [22]. Weiterhin sind bei Ratten mit Leberzirrhose systemische Plasmaspiegel von NA höher als in PVL-Ratten. Dies zeigt, dass eine erhöhte Aktivität des SNS teils durch die Entwicklung einer Zirrrhose bedingt ist, und nicht lediglich durch Induktion einer experimentellen prähepatischen portalen Hypertension (mittels Portal-Venen-Ligatur) erreicht werden kann [5].

Protokoll II: Anhaltende Effekte der vaskulären Desensibilisierung und der Einfluss von exogenem NPY. Um die langandauernden Effekte der Degradation der vaskulären Druckantwort im 1. Perfusionszyklus zu untersuchen, wurde das Präparat anschließend in einer „Auswaschphase“ für 1 Stunde mit Krebspuffer perfundiert. Danach erfolgte ein 2. Perfusionszyklus (2. PC) mit je 5 Stimulationen (genannt PNS-1b bis PNS-5b). Die gesamte Versuchsdauer betrug ca. 3,7- 3,9 Stunden. Um den Effekt von exogenem NPY zu untersuchen, wurden die Gruppen aufgeteilt [Con: $n=8$, Con+NPY: n=8, PVL: n=6, PVL+NPY: n=6, LC: n=11, LC+NPY: $n=11]$. In den NPY-Gruppen wurde nach der Hälfte der einstündigen Auswaschphase exogenes NPY in einer Konzentration von 50nM zur Perfusionslösung zugesetzt bis zum Ende des 2. PC. In vorherigen Arbeiten konnten wir zeigen, dass exogenes 50nM NPY per se nicht direkt vasokonstriktiv wirkt. Für die Messung der NASekretion wurden analog zu Protokoll I erneut Proben in einer weiteren Versuchsreihe gesammelt [Con: $n=12$, LC: $n=12$ ]. Während des 2. Perfusionszyklus (2. PC) wurde die Freisetzung von endogenem NPY nicht bestimmt, da das exogen zugeführte NPY hier eine zulässige Interpretation verhindern würde.

Statistische Auswertung. Wir verwendeten den Kolmogorov-Smirnov-Test zur Überprüfung von Normalverteilungen. Je nach Notwendigkeit wurde zum Vergleich der Mittelwerte eine Varianzanalyse oder der gepaarte oder ungepaarte t-Test herangezogen. Das Signifikanzniveau betrug $\alpha<0.05$. Für sämtliche statistischen Berechnungen sowie zur graphischen Darstellung der Ergebnisse wurde das Programm IBM SPSS Statistics 19 verwendet. 


\section{c. Ergebnisse}

Tiere. In portal hypertensiven Tiergruppen (PVL und LC) zeigte sich ein signifikant erhöhtes absolutes und relatives Milzgewicht (Tabelle 1). Dabei korrelierte das Milzgewicht als Parameter für das Ausmaß portaler Hypertension signifikant mit der mittleren vaskulären Druckantwort auf periarterielle Nervenstimulation (PNS) $(r=-0.86, p<0.01)$ und zeigte einen negativ logarithmischen Verlauf $\left(\mathrm{R}^{2}=0.8\right.$, siehe auch Supplementary Material, Suppl.-Fig. 1).

\begin{tabular}{|c|c|c|c|}
\hline \multirow[t]{2}{*}{ Tabelle 1} & \multicolumn{3}{|c|}{ Basalwerte und hämodynamische Parameter [1. Perfusionszyklus] } \\
\hline & Con & PVL & $\mathbf{L C}$ \\
\hline Milzgewicht/Körpergewicht [g/kg KG] & $1.7 \pm 0.3$ & $4.2 \pm 1.0 * * *$ & $6.0 \pm 0.9 * * *$ \\
\hline Milzgewicht/mittl. Druckantwort 1. PC [g/mmHg] & $0.005 \pm 0.001$ & $0.019 \pm 0.005^{* * *}$ & $0.044 \pm 0.014 * * *$ \\
\hline Baseline $[\mathrm{mmHg}]$ & $21.4 \pm 6.3$ & $15.4 \pm 3.3^{*}$ & $16.3 \pm 4.1^{*}$ \\
\hline Mittlere Druckantwort=PR [mmHg] & $114.3 \pm 17.5$ & $74 \pm 9.5 * * *$ & $57.4 \pm 11.3 * * *$ \\
\hline Abfall der PR [\%] & $34.6 \pm 17.1$ & $55.1 \pm 10.9 * * *$ & $50.4 \pm 8.1 * * *$ \\
\hline DP-40 & $12.8 \pm 2.3$ & $9.8 \pm 2.8 * *$ & $10.2 \pm 3 * *$ \\
\hline
\end{tabular}

DP-40 = Anzahl der PNS zur Induktion eines 40\%-igen Abfalls der Druckantwort . $* \mathrm{p}<0.05$ vs. Con $* * \mathrm{p}<0.01$ vs. Con $* * * \mathrm{p}<0.001$ vs. Con

Repetitive periarterielle Nervenstimulation (PNS) und vaskuläre Druckantwort im 1. Perfusionszyklus (1. PC). Der basale Perfusionsdruck (=Baseline) war signifikant geringer in portal hypertensiven Tieren (Tabelle 1). Die vaskuläre Druckantwort (pressure-response $=P R$ ) erreichte immer bei PNS-3 den Maximalwert und fiel danach sukzessive bei jeder weiteren PNS ab. Diesen Prozess bezeichnen wir hier als Desensibilisierung. Bei portaler Hypertension kam es generell zu einer signifikant niedrigeren Druckantwort (Abb. 2A). Die Degradation der Druckantwort (PR) wurde anhand des Abfalls in Relation zur maximalen PR (bei PNS-3) bewertet und dargestellt. Dieser Degradations- oder Desensibilisierungs-Prozess war bei den portal hypertensiven Tiergruppen stärker ausgeprägt und der Abfall verlief schneller (Abb. 2B). So waren bei portaler Hypertension signifikant weniger repetitive PNS notwendig, um beispielsweise einen 40\%-Abfall der maximalen Druckantwort zu erreichen (Tabelle 1). 

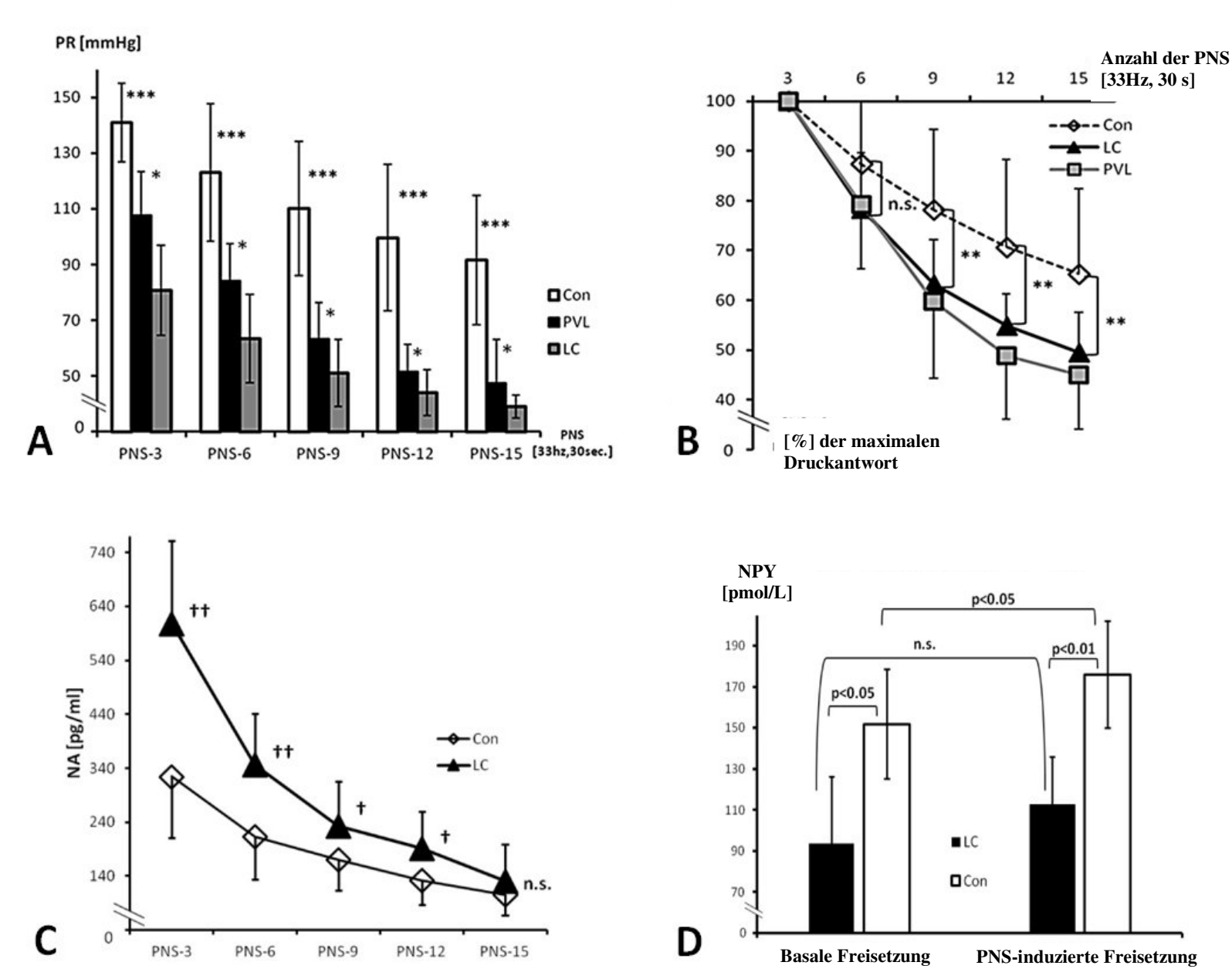

Abbildung 2: Druckantwort (pressure-response=PR) und Neurotransmitter Freisetzung im 1. Perfusionszyklus. $\underline{A}$ : Die PNS-induzierte Druckantwort war bei portaler Hypertension signifikant vermindert [PVL=n=12, LC: $\mathrm{n}=19$, Con: $\mathrm{n}=19]$ ] $\underline{B}$ : Gesteigerter Druckabfall bei repetitiver Nervenstimulation bei PVL und LC. $\underline{C}$ : Die PNS-induzierte NA-Freisetzung war bei LC $(\mathrm{n}=12)$ erhöht im Vergleich zu gesunden Kontrolltieren $(\mathrm{n}=12)$. D: Die basale und PNS-induzierte Sekretion von NPY hingegen war bei LC signifikant vermindert. *: $\mathrm{p}<0.05$ vs. LC; $* *: \mathrm{p}<0.01$; ***: $\mathrm{p}<0.001$ vs. PVL und LC; $\uparrow: \mathrm{p}<0.05$ vs. Con; $\dagger \uparrow: \mathrm{p}<0.01$ vs. Con. Con, Kontrolltiere. LC, Leberzirrhose. NA, Noradrenalin. PC, Perfusionszyklus. PNS, periarterielle Nervenstimulation. PVL, Portal-Venen-Ligatur.

PNS-induzierte NA-Freisetzung und Druckantwort (PR) im 1. PC. Erwartungsgemäß war die basale NA-Sekretion in beiden Gruppen sehr gering (Con: $13.6 \pm 9.6 \mathrm{pg} / \mathrm{ml}$ vs. LC: $15.0 \pm 6.2$ pg/ml; n.s.). Die durch PNS induzierbare NA-Sekretion war höher bei zirrhotischen Tieren (Abb. 2C). Mit steigender Anzahl der PNS nahm die jeweilige Sekretion von Noradrenalin sukzessive ab. Diese Abnahme der NA-Sekretion war bei Leberzirrhose (477.5 $\pm 121.6 \mathrm{pg} / \mathrm{ml})$ im Vergleich zu gesunden Kontrolltieren $(219.9 \pm 110.9 \mathrm{pg} / \mathrm{ml} ; \mathrm{p}<0.001)$ signifikant stärker ausgeprägt. Um die Effizienz von NA bezüglich vasokonstriktiver Wirkung abschätzen zu können, berechneten wir das Verhältnis von NA-Sekretion zur jeweiligen vaskulären Druckantwort (PR). Je mehr NA also für eine Druckantwort nötig ist, desto höher ist diese Ratio NA-Sekretion/PR. Tatsächlich zeigten sich die NA/PR-Ratios bei zirrhotischen Ratten stark erhöht (LC: $5.7 \pm 2.2[\mathrm{pg} / \mathrm{ml}] / \mathrm{mmHg}$ vs. Con: $1.7 \pm 0.5[\mathrm{pg} / \mathrm{ml}] / \mathrm{mmHg} ; \mathrm{p}<0.01)$. 

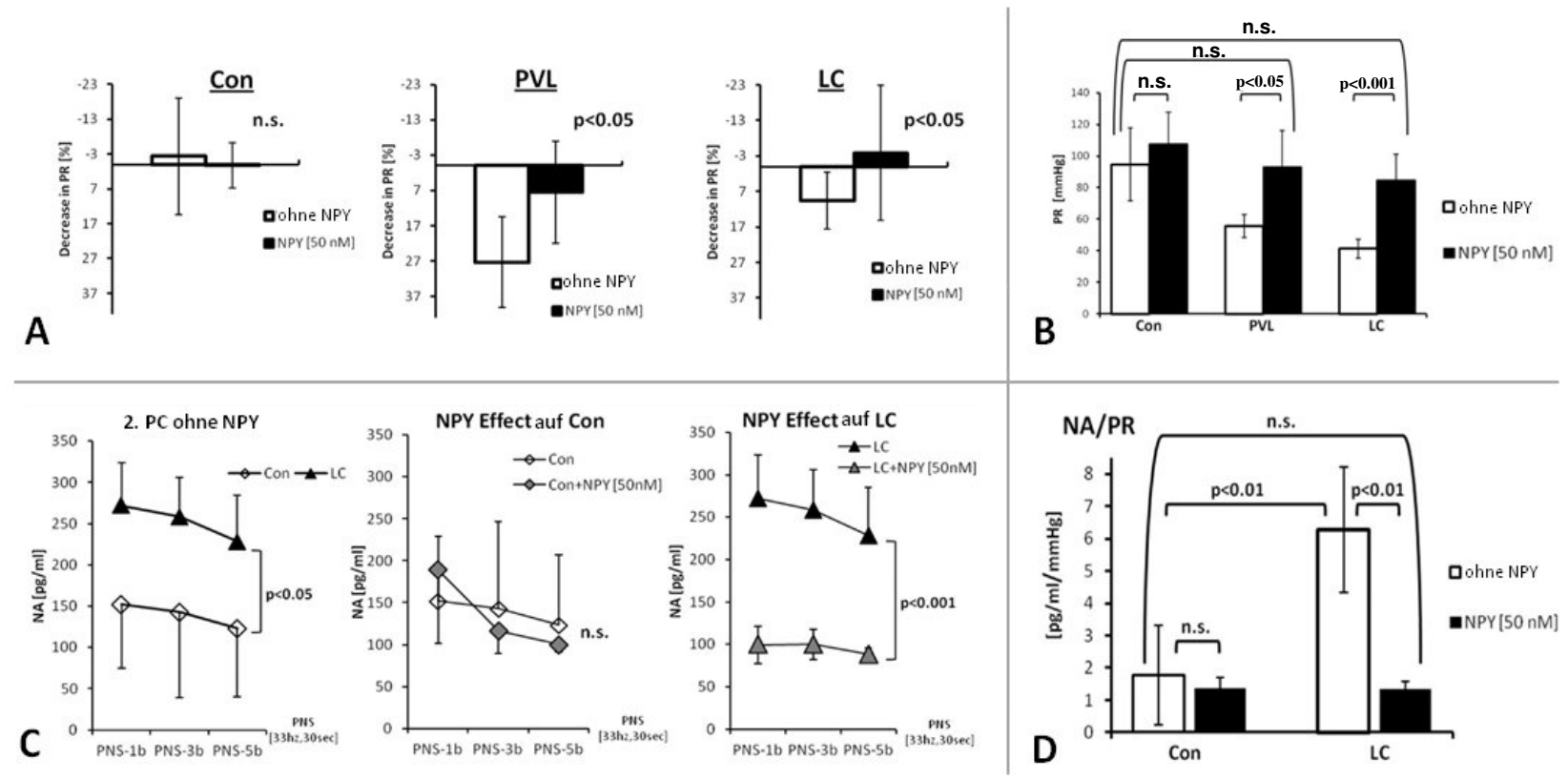

Abbildung 3: Druckantwort (PR), NA-Sekretion und der Einfluss von exogenem NPY im 2. PC. Einfluss von exogenem NPY $[50 \mathrm{nM}]$ auf die PNS-induzierte Druckantwort $\underline{(B)}$ und der Abfall von PR bei repetitiver Nervenstimulation im 2. PC $\underline{(A)}$. Con: n=8, Con+NPY: n=8, PVL: n=6, PVL+NPY: n=6, LC: n=11, LC+NPY: n=11. $C$ : PNS-induzierte NA-Freisetzung und Einfluss von exogenem NPY [Con: $n=6$, Con+NPY: $n=6$, LC: $n=6$, LC+NPY: $n=6$ ]. $\underline{D}$ : Das Verhältnis von NA-Freisetzung zu Druckantwort (PR) war bei Zirrhose wie im 1. PC erhöht. In Anwesenheit von exogenem NPY war die bei LC-Ratten pathologisch gesteigerte Ratio NA/PR deutlich reduziert. Con, Kontrolltiere. LC, Leberzirrhose. NA, Noradrenalin. PC, Perfusionszyklus. PNS, periarterielle Nervenstimulation. PVL, Portal-Venen-Ligatur.

PNS-induzierte NPY-Sekretion im 1. PC. Sowohl die basale NPY-Freisetzung als auch die PNS-induzierbare NPY-Sekretion war bei Leberzirrhose vermindert (Abb. 2D). Im Kontrast zum sukzessiven Abfall der PNS-induzierten NA-Sekretion nahm die NPY-Freisetzung während der repetitiven PNS nicht ab, somit war die Menge des freigesetzten endogenen NPY unabhängig von der Anzahl der Nervenstimulationen. Verglichen mit der basalen Sekretion, konnte durch periarterielle Nervenstimulation nur bei gesunden Tieren eine erhöhte Freisetzung erzielt werden $(A b b .2 D)$. Betrachtet man nun das Verhältnis der Sekretion von Transmitter NA und Ko-Transmitter NPY, so zeigte sich bei zirrhotischen Tieren eine niedrigere NPY/NARatio $(0.6 \pm 0.2[\mathrm{pmol} / \mathrm{L}] /[\mathrm{pg} / \mathrm{ml}])$ als bei Kontrolltieren $(1.2 \pm 0.3[\mathrm{pmol} / \mathrm{L}] /[\mathrm{pg} / \mathrm{ml}], \mathrm{p}<0.001$; siehe auch Supplementary Material, Suppl.-Fig. 4B). Dies zeigt neben dem absoluten Mangel an NPY auch eine im Vergleich zur erhöhten NA-Freisetzung verminderte relative Sekretion des Ko-Transmitters NPY im Splanchnikusgebiet bei Leberzirrhose. 
Druckantwort und Einfluss von exogenem NPY im 2. Perfusionszyklus (2. PC). Während der Auswaschphase und während des 2. PC war der basale Perfusionsdruck wie im 1. PC niedriger in PVL- und LC-Ratten $(\mathrm{p}<0.05)$ und wurde durch das der Perfusionslösung zugesetzte exogene NPY nicht beeinflusst. Die PNS-induzierbare Druckantwort (PR) war erneut niedriger bei portal hypertensiven Tieren (Abb. 3B). Der Abfall der Druckantworten während des 2. PC war nur bei portal hypertensiven Tieren signifikant und wurde in Gegenwart von exogenem NPY vermindert $(A b b .3 A)$. Exogenes NPY erhöhte die PNS-induzierbare Druckantwort in Kontrolltieren geringfügig. Bei PVL- und LC-Ratten jedoch kam es zu einer stark gesteigerten Druckantwort $(A b b .3 B)$. Um das Ausmaß der zeitabhängigen "Erholung” der vaskulären Druckantwort nach der einstündigen Auswaschphase beurteilen zu können und somit ein Maß für die langanhaltenden Effekte des Desensibilisierungs-Prozesses im 1. Perfusionszyklus zu erhalten, verglichen wir die erste Druckantwort des 2. PC [PNS-1b] mit der letzten Druckantwort des 1. PC [PNS-15].

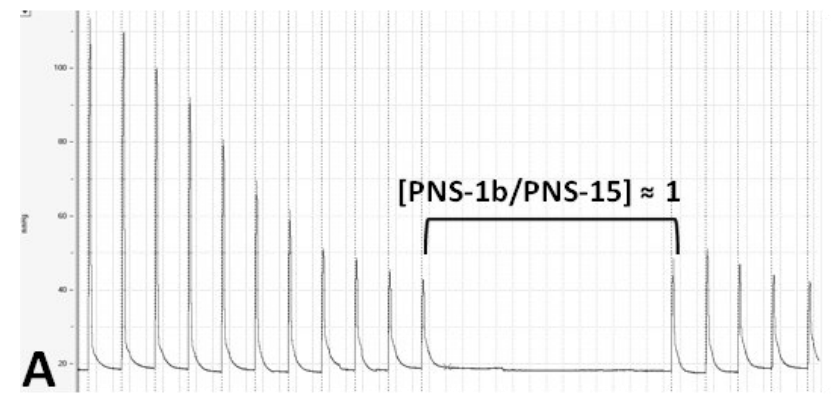

Con

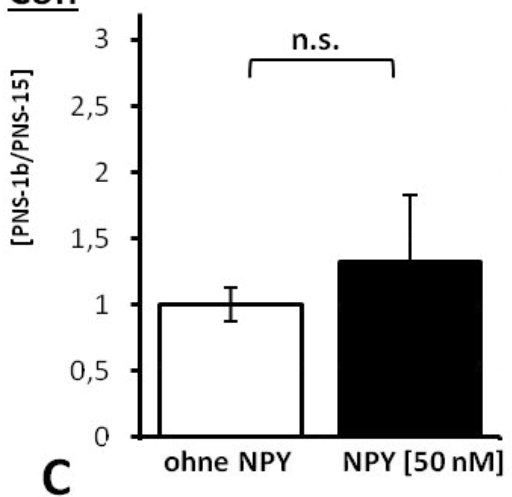

$\underline{\text { PVL }}$

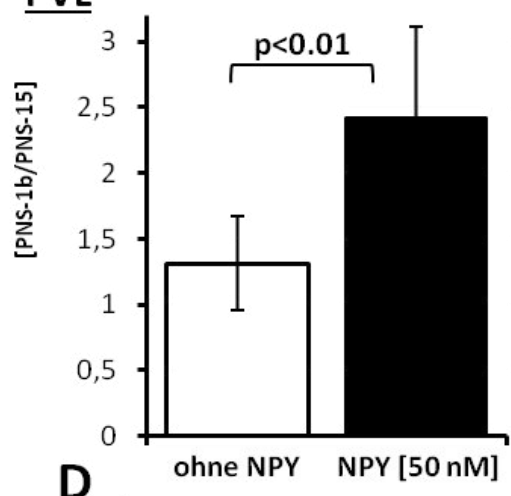

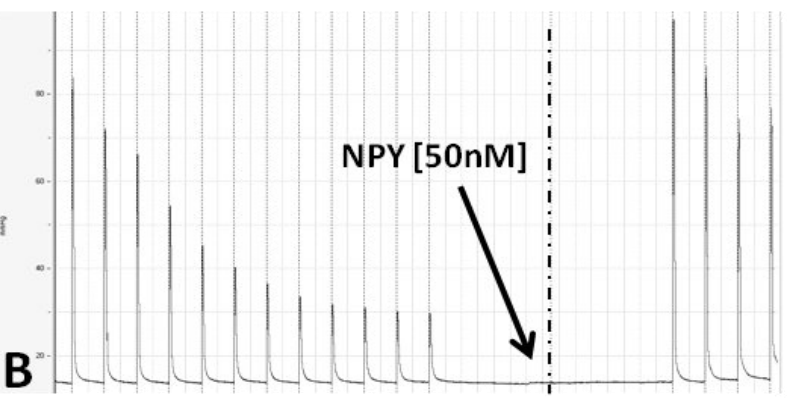

$\underline{\underline{L C}}$

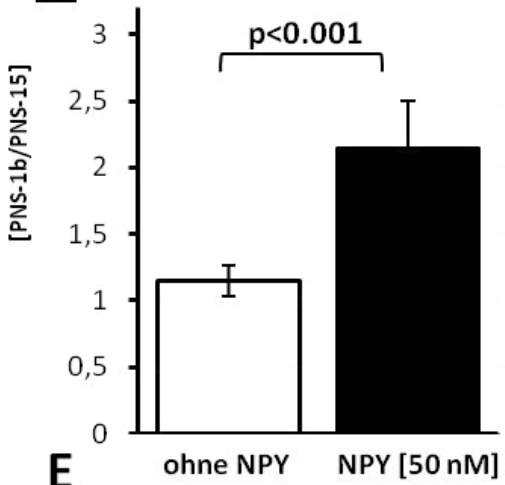

Abbildung 4: „Erholung“ der PNS-induzierten Druckantwort mit und ohne exogenem NPY. A: Der repräsentative Ausschnitt zeigt die durch repetitive PNS induzierten vaskulären Druckantworten des 1. Perfusionszyklus (PC), gefolgt von einer einstündigen Auswaschphase und einem 2. PC mit repetitiven PNS. $\underline{B}$ : Erholung der Druckantwort bei einer zirrhotischen Ratte in Anwesenheit von 50nM NPY. Ohne exogenes NPY war in keiner Gruppe eine wesentliche Erholung der PNSinduzierten Druckantwort zu sehen $(C-E)$. Bei gesunden Kontrolltieren führte exogenes NPY zu keiner signifikanten Erhöhung der Ratio [PNS-1b/PNS-15] ( $\underline{C}$. In portal hypertensiven Tieren wurde diese Ratio durch exogenes NPY signifikant erhöht ( $\underline{B}$, $\underline{D, E}$. Con: $\mathrm{n}=8$; Con+NPY: n=8; PVL: $\mathrm{n}=6$; PVL+NPY: $\mathrm{n}=6$; LC: $\mathrm{n}=11 ; \mathrm{LC}+\mathrm{NPY}: \mathrm{n}=11$. Con, Kontrolltiere. LC, Leberzirrhose. NA, Noradrenalin. PC, Perfusionszyklus. PNS, periarterielle Nervenstimulation. PVL, Portal-Venen-Ligatur. 
Somit stellt die Ratio [PNS-1b/PNS-15] einen Indikator für die Wiederherstellung der Fähigkeit der mesenterialen arteriellen Gefäße zur Vasokonstriktion nach einer einstündigen Auswaschphase dar $(A b b .4 A)$. Ohne exogenes NPY war in keiner Gruppe eine wesentliche Erholung der vaskulären PNS-induzierten Druckantwort zu sehen (Abb. $4 C-E)$. Bei gesunden Kontrolltieren führte exogenes NPY zu keiner signifikanten Erhöhung der Ratio [PNS-1b/PNS15] $(A b b .4 C)$. In portal hypertensiven Tieren wurde diese Ratio durch exogenes NPY signifikant erhöht und die vaskuläre Druckantwort wiederhergestellt ( $A b b .4 B, D, E)$. So verursachte exogenes NPY eine 32.5 $550.1 \%$-ige Erhöhung der Druckantwort auf periarterielle Nervenstimulation bei gesunden Ratten (n.s.) und eine 114.3 $\pm 35.5 \%$-ige Steigerung bei LCRatten $(\mathrm{p}<0.001)$.

NA-Sekretion und der Einfluss von exogenem NPY im 2. PC. Wie im 1. PC zeigten zirrhotische Ratten erneut eine erhöhte PNS-induzierte NA-Freisetzung. Exogenes NPY verminderte die NA-Freisetzung in gesunden Tieren geringfügig. Bei zirrhotischen Tieren jedoch war die PNS-induzierte NA-Sekretion in der Gegenwart von exogenem NPY stark vermindert $(A b b .3 C)$. Das Verhältnis von NA-Freisetzung zu Druckantwort (PR) war bei Zirrhose wie im 1. PC mehr als 3-fach erhöht. In Anwesenheit von exogenem NPY war die bei LC-Ratten pathologisch gesteigerte Ratio NA/PR deutlich reduziert und unterschied sich nicht mehr signifikant von den Werten bei gesunden Tieren (Abb. 3D). 


\section{d. Diskussion}

Viele Studien erbrachten bislang Hinweise auf eine gesteigerte Aktivität des sympathischen Nervensystems (SNS) bei Leberzirrhose [3, 6-9]. In letzter Zeit vermehren sich jedoch Anhaltspunkte dafür, dass bei portaler Hypertension eine splanchnische Atrophie des SNS zu vermehrter mesenterialer arterieller Vasodilatation beitragen könnte. So wurde in mesenterialen Arterien bei portaler Hypertension eine ausgeprägte Reduktion von sympathischen Nerven sowie eine verminderte Expression der Tyrosin-Hydroxylase - einem Schlüssel-Enzym der Noradrenalin-Synthese - nachgewiesen [10-12]. Unter Verwendung von repetitiven elektrischen sympathischen Nervenstimulationen und somit Nachahmung einer anhaltenden und exzessiven Aktivität des SNS im Splanchnikusgebiet bei Ratten mit Leberzirrhose konnten wir eine im Durchschnitt um 30\% erhöhte NA-Freisetzung nachweisen. Initial war die NASekretion bei Zirrhose sogar annähernd doppelt so hoch als bei gesunden Tieren. Obwohl die erhöhte Noradrenalin-Sekretion auf den ersten Blick in Wiederspruch $\mathrm{zu}$ den oben beschriebenen Studien stehen, welche eine reduzierte SNS-Aktivität im Splanchnikusgebiet bei portaler Hypertension aufzeigen, könnte diese Diskrepanz durch periphere Zellen des Immunsystems, welche mit Katecholamin-produzierenden Enzymen ausgestattet sind, erklärt werden [23]. Es gibt sogar indirekte Hinweise darauf, dass Immunzellen durch elektrische Stimulation zur Freisetzung von Neurotransmittern angeregt werden können [24]. Bei vielen chronisch-inflammatorischen Erkrankungen kommt es zu einem Verlust sympathischer Nervenfasern [25], und dieser Verlust geht mit einer gleichzeitigen Vermehrung von TyrosinHydoxylase positiven Immunzellen einher [26]. Bei Leberzirrhose mit Aszites kommt es bekannterweise zu einer chronischen "low-level" Inflammation, und somit könnte man spekulieren, ob vielleicht eine gesteigerte Infiltration des Splanchnikusgebietes mit Katecholamin-produzierenden Immunzellen $\mathrm{zu}$ einer erhöhten PNS-induzierbaren NASekretion beitragen könnte. Wie dem auch sei, diese Hypothesen waren nicht Bestandteil dieser Studie und sollten demnach im Rahmen weiterer Untersuchungen adressiert werden. Nichtsdestoweniger zeigen unsere Daten Übereinstimmungen mit indirekten Hinweisen auf eine gesteigerte splanchnische NA-Sekretion: Die Noradrenalin-Konzentration zeigte sich im Portalvenenblut bei humaner [6] und experimenteller [5] Leberzirrhose erhöht im Vergleich zu arteriellen und/oder peripher-venösen Blutproben. Dazu in Kontrast stehend fanden wir eine verminderte PNS-induzierte splanchnische NPY-Sekretion bei zirrhotischen Ratten. Des Weiteren zeigte sich eine im Vergleich zur basalen NPY-Sekretionsrate gesteigerte NPYFreisetzung als Folge einer elektrischen Nervenstimulation nur bei gesunden Tieren. 
Dies könnte neben einer verminderten basalen Freisetzungsrate auch auf eine verminderte Fähigkeit der splanchnischen sympathischen Nervenfasern zur stimulierten NPY-Sekretion bei portaler Hypertension hindeuten. Außerdem könnte die verminderte Freisetzung von NPY das funktionelle Korrelat zur oben beschriebenen Regression sympathischer Nervenaktivität im mesenterialen arteriellen Gefäßbett bei Leberzirrhose darstellen [10, 11]. Neben der lokal verminderten SNS-Aktivität gibt es weitere potenzielle Mechanismen zur Erklärung eines NPY-Mangels: Zweifellos ist die systemische - und in dieser Studie auch die lokale, mesenterial freigesetzte - Noradrenalin-Konzentration bei Zirrhose erhöht [3, 6-9]. Da bei intaktem Kreislauf diese erhöhten NA-Konzentrationen auch das splanchnische arterielle Gefäßbett erreichen, könnte dies zu einer gesteigerten präsynaptischen Hemmung der NPYFreisetzung via $\alpha_{2}$-Rezeptoren führen [27, 28]. Tatsächlich führt eine wiederholte Stimulation sympathischer Nervenfasern zu einer Abnahme der NPY-Freisetzung [29]. Eine weitere Erklärung für eine lokal verminderte splanchnische NPY-Wirkung könnte die bei Leberzirrhose gesteigerte Aktivität der Dipeptidyl-Peptidase-IV (DPP-IV) sein [30]. Die DPP-IV ist Bestandteil des extrazellulären Protein-Metabolismus und ist in unmittelbarer Nähe von endothelialen und glattmuskulären Oberflächenrezeptoren lokalisiert [31]. Dabei ist die DPP-IV unter anderem an der Prozessierung von NPY beteiligt [32]. Folglich kann die durch gesteigerte DPP-IV-Aktivität vermehrte Prozessierung von NPY bei Zirrhose zu vermehrter Bildung von NPY-Spaltprodukten führen, was dann in einer verminderten Stimulation des postsynaptischen, Vasokonstriktion vermittelnden, $\mathrm{Y}_{1}$-Rezeptors resultieren könnte. In dieser Studie fanden wir bei Zirrhose eine im Splanchnikusgebiet (vor allem initial stark) vermehrte NA-Sekretion bei gleichzeitig signifikant verminderter NPY-Freisetzung. Diese Dysbalance in der SNSNeurotransmitter Freisetzung könnte auch durch Endotheline (ET-1, ET-3) erklärt werden, welche zwar die NPY-Sekretion inhibieren, dabei aber die NA-Freisetzung nicht beeinflussen [33]. Interessanterweise sind zirkulierende Plasmaspiegel von ET-1 und ET-3 bei Leberzirrhose erhöht [34, 35], und könnten somit zu einer selektiven Inhibition der NPY-Sekretion beitragen. Die beobachtete Dysregulation der NA-Freisetzung hingegen, insbesondere die schnellere und stärkere Depletion der PNS-induzierten NA-Sekretion bei Zirrhose, könnte die Auswirkung einer verminderten Expression von Genen sein, welche den axonalen und vesikulären Transport, sowie die Exozytose von Katecholaminen regulieren [11]. Somit könnte eine Atrophie sympathischer Nervenfasern initial zu einer kompensatorisch gesteigerten NAFreisetzung aus den verbliebenen Nervenendigungen führen, jedoch dabei mit einem schnelleren Verlust von präsynaptisch gespeichertem NA und damit schnelleren Abfall der NA-Freisetzung bei repetitiver PNS einhergehen. 
Die hier verminderte lokale NPY-Freisetzung im Splanchnikusgebiet steht im Gegensatz zu erhöhten systemischen NPY-Plasmaspiegeln bei Patienten mit Leberzirrhose [36]. Nichtsdestotrotz reflektieren systemische Plasmaspiegel lediglich ein "spill-over" der Neurotransmitter aus dem synaptischen Spalt, welche von den unterschiedlichsten Kompartimenten des Organismus in die systemische Zirkulation abgegeben werden. Systemische NPY-Plasmaspiegel sind somit das Resultat von Produktion, Sekretion, Degradation und Metabolismus im gesamten Organismus [4]. Unterschiede zwischen lokaler und systemischer NPY-Produktion und Sekretion schließen sich daher keinesfalls aus. Hier zeigten portal hypertensive Ratten nicht nur die bereits bekannte vaskuläre Hyporeaktivität [14, 15, 16], sondern zudem einen gesteigerten Abfall der vaskulären PNS-induzierten Druckantwort. Dieser Desensibilisierungs-Prozess scheint in gewissem Maße physiologisch zu sein, da er auch bei den gesunden Tieren zu sehen war - das Ausmaß jedoch war bei portaler Hypertension erhöht. Bei einer Synopsis unserer Ergebnisse für Druckantworten und gleichzeitiger Neurotransmitter-Sekretion lässt sich erkennen, dass die Hypothese einer postsynaptischen „Desensibilisierung der glatten Gefäßmuskelzelle“ erweitert werden sollte, da es scheint, als wäre der gesteigerte Abfall der Druckantwort bei anhaltender SNS-Aktivität zumindest teilweise auch auf einen schnelleren und gesteigerten Abfall der präsynaptischen NA-Freisetzung sowie auf eine mangelhafte NPY-Sekretion zurückzuführen. Dies stimmt auch mit der Feststellung überein, dass die vaskuläre Hyporeaktivität bei Leberzirrhose nicht durch eine veränderte Anzahl oder Affinität der postsynaptischen $\alpha_{1}$-Adrenorezeptoren bedingt ist [37]. Da NPY ein potenter „Verstärker“ und Modulator der vasokonstriktiven Wirkung von NA ist [13-16], scheint es, als sei nicht nur die absolute Menge der NPY-Sekretion relevant, sondern vielmehr das Verhältnis von Transmitter und Ko-Transmitter. Die hier bei Zirrhose veränderten Proportionen von NA/NPY und NA/Druckantwort unterstreichen diese Hypothese. Der beobachtete Abfall der NA-Freisetzung bei gleichzeitig stabilerer und durch PNS weniger beeinflussbarer basaler splanchnischer NPY-Sekretion könnte die Vermutung nahelegen, dass NPY eine nervale vasokonstriktive Reserve darstellt, welche in Situationen mit exzessiver Sympathikus-Aktivierung, wie beispielsweise bei gastrointestinaler Blutung, den überproportionalen Verlust bzw. Verbrauch von NA teilweise kompensieren kann. Allem Anschein nach ist diese vaskuläre Reservefunktion im mesenterialen Gefäßbett bei Zirrhose weitgehend limitiert.

Knapp 30 Prozent der Sympathikus-vermittelten splanchnischen Vasokonstriktion werden durch NPY vermittelt [20]. Dies untermauert die klinisch-pathologische Signifikanz der hier gezeigten verminderten NPY-Sekretion im Splanchnikusgebiet. Zudem könnte der lokale NPY- 
Mangel einige unserer vorherigen Ergebnisse erklären, vor allem die Frage, warum die folgenden Effekte von exogenem NPY bei portaler Hypertension viel ausgeprägter sind als unter physiologischen Bedingungen: NPY i) hemmt die eNOS- und nNOS-vermittelte Produktion von NO und dessen vasodilatierende Wirkung [16, 38], ii) erhöht die Expression und Aktivität der vasokonstriktiven Rho-A-Kinase [38] und iii) reduziert den Portalvenendruck und den portalvenösen Blutfluss [38]. In dieser Studie korrigierte exogenes NPY nicht nur die PNS-induzierte Druckantwort mesenterialer Arterien bei portaler Hypertension, sondern verlangsamte auch den Prozess der sukzessiven Degradation der Druckantwort und konnte zudem die vaskuläre Sensitivität für endogenes NA im 2. Perfusionszyklus wiederherstellen. Die NPY-induzierte Potenzierung $\alpha_{1}$-adrenerger Vasokonstriktion via postsynaptischer $\mathrm{Y}_{1^{-}}$ Rezeptoren ist bekannt [13, 14, 38]. Daneben wurden die präsynaptischen Effekte von NPY bereits bei gesunden Ratten untersucht: NPY vermittelt einen negativen FeedbackMechanismus an der präsynaptischen Membran via $\mathrm{Y}_{1^{-}}, \mathrm{Y}_{2^{-}}, \mathrm{Y}_{5^{-}}$und weiterer Rezeptoren und limitiert dadurch eine weitere NA-Freisetzung [39]. In dieser Arbeit konnten wir zeigen, dass NPY die Freisetzung von NA bei zirrhotischen Tieren um ein vielfaches stärker hemmt als bei gesunden Tieren. Die oben beschriebene Ratio von NA/Druckantwort, welche einen Indikator für die NA-Menge darstellt, die zu einem bestimmten Maß an Vasokonstriktion führt, war bei zirrhotischen Ratten unter dem Einfluss von exogenem NPY viel stärker vermindert als bei gesunden Tieren. Dies zeigt die große Potenz von exogenem NPY, die vasokonstriktive Sensitivität für NA bei Zirrhose zu erhöhen. Die verstärkten post- und präsynaptischen Effekte von NPY bei portaler Hypertension passen auch zu der von uns bereits gezeigten erhöhten Produktion und Aktivität von nNOS, eNOS, und NO bei Zirrhose, da deren Überproduktion bei kranken Tieren zu einer entsprechend stärkeren durch NPY induzierbaren Aktivitäts-Hemmung und dadurch stärkeren Reduktion der Vasodilatation führen könnte [40, 41]. Kolo et al. zeigten, dass der vasokonstriktive Effekt von $N A$ durch NO abgeschwächt wird, die vasokonstriktive Wirkung von NPY jedoch von NO unbeeinflusst bleibt [42]. Somit ist die gesteigerte NPYWirkung bei Zirrhose in unseren Experimenten bei intaktem Endothel und damit erhöhter NOProduktion plausibel. Weiterhin zeigten wir, dass das Hitze-Schock-Protein-90 (HSP-90) in mesenterialen Arterien ein Signalmediator der nNOS- und eNOS-bedingten Vasodilatation ist und dass die NO-abhängige Vasorelaxation bei portaler Hypertension durch HSP-90 vermittelt

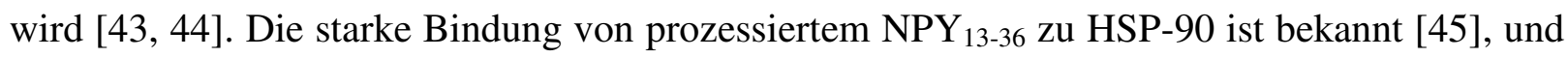
könnte auf einen Mechanismus der NPY-vermittelten Regulation oder Modulation von NOS und NO hindeuten. Proteolytische Prozessierung von NPY kann über das Spaltprodukt NPY 3-36 $\mathrm{zu}$ einer selektiven Aktivierung des präsynaptischen $Y_{2}$-Rezeptors führen [32]. 
In diesem Zusammenhang könnte der durch exogenes NPY induzierte $\mathrm{Y}_{2}$-Rezeptor-vermittelte Abfall der NA-Sekretion bei Leberzirrhose deshalb stärker sein, weil die oben erwähnte, bei Zirrhose erhöhte DPP-IV-Aktivität [30] zu einer gesteigerten proteolytischen Prozessierung von NPY und damit gesteigerten präsynaptischen Stimulation des $\mathrm{Y}_{2}$-Rezeptor und dadurch zu vermehrter Inhibition der NA-Sekretion führt. Darüber hinaus wurde auch eine cholinerge $M_{3^{-}}$ Rezeptor abhängige Vasodilatation im perfundierten mesenterialen Gefäßbett der Ratte beschrieben [46]. Interessanterweise wirkt NPY über $\mathrm{Y}_{2}$-Rezeptoren inhibierend auf die sympathische cholinerge Vasodilatation, aber nicht auf die sympathische adrenerge Vasokonstriktion [47]. Dies könnte einen weiteren Mechanismus darstellen, wie exogenes NPY über eine spezifische Bindung an den $\mathrm{Y}_{2}$-Rezeptor die gesteigerte Vasodilatation bei Zirrhose hemmen kann und sollte ebenfalls in zukünftigen Studien untersucht werden.

Zusammenfassend stellen wir ein Ungleichgewicht der mesenterialen sympathischen Neurotransmitter-Freisetzung - genauer gesagt eine gesteigerte NA-Sekretion bei gleichzeitig verminderter NPY-Sekretion - bei zirrhotischen Ratten fest, und daraus resultierend eine vasokonstriktive Hyporeaktivität (Abb. 5A). Exogenes NPY hat dabei wenig Effekt auf die vaskuläre Druckantwort bei gesunden Tieren. Bei portaler Hypertension hingegen verstärkt NPY die PNS-induzierte Druckantwort, reduziert den vaskulären Desensibilisierungs-Prozess und vermindert die pathologisch gesteigerte NA-Sekretion signifikant und kann dadurch wahrscheinlich die bei Zirrhose gesteigerte NA-Freisetzung ökonomisieren. Diese Ergebnisse weisen sowohl auf eine prä- als auch postsynaptisch gesteigerte Wirkung von NPY auf portal hypertensive splanchnische Arterien hin (Abb. 5B).

Im menschlichen Organismus ist die splanchnische Vasokonstriktion mit der lokalen Freisetzung von NA und NPY assoziiert. Eine zusätzliche Erhöhung der NPY- und Katecholamin-Plasmaspiegel nach Propranolol-Gabe kommt dabei wahrscheinlich durch eine gesteigerte SNS-Aktivität und/oder verminderten Abbau der Transmitter zustande [48]. Dies zeigt, dass NPY auch eine Rolle bei der splanchnischen Wirkung von non-selektiven $\beta$-Blockern bei Leberzirrhose spielen könnte. Bei gesunden Menschen wurde gezeigt, dass eine intravenöse NPY-Infusion (i) den splanchnischen Blutfluss stark reduziert und damit dem hyperdynamen Zirkulationssyndrom bei Leberzirrhose entgegenwirken könnte, (ii) den renalen Blutfluss leicht reduziert und (iii) interessante metabolische Wirkungen hervorruft, wie die Hemmung splanchnischer Glykogenolyse oder die Regulation der Nahrungsaufnahme und des Appetits $[49,50]$. Aus diesen Gründen sollte die Infusion von NPY bei Patienten mit portaler Hypertension unter strenger Kontrolle renaler und metabolischer Marker getestet werden, um die potenziellen splanchnischen hämodynamischen Effekte bei Leberzirrhose zu untersuchen. 

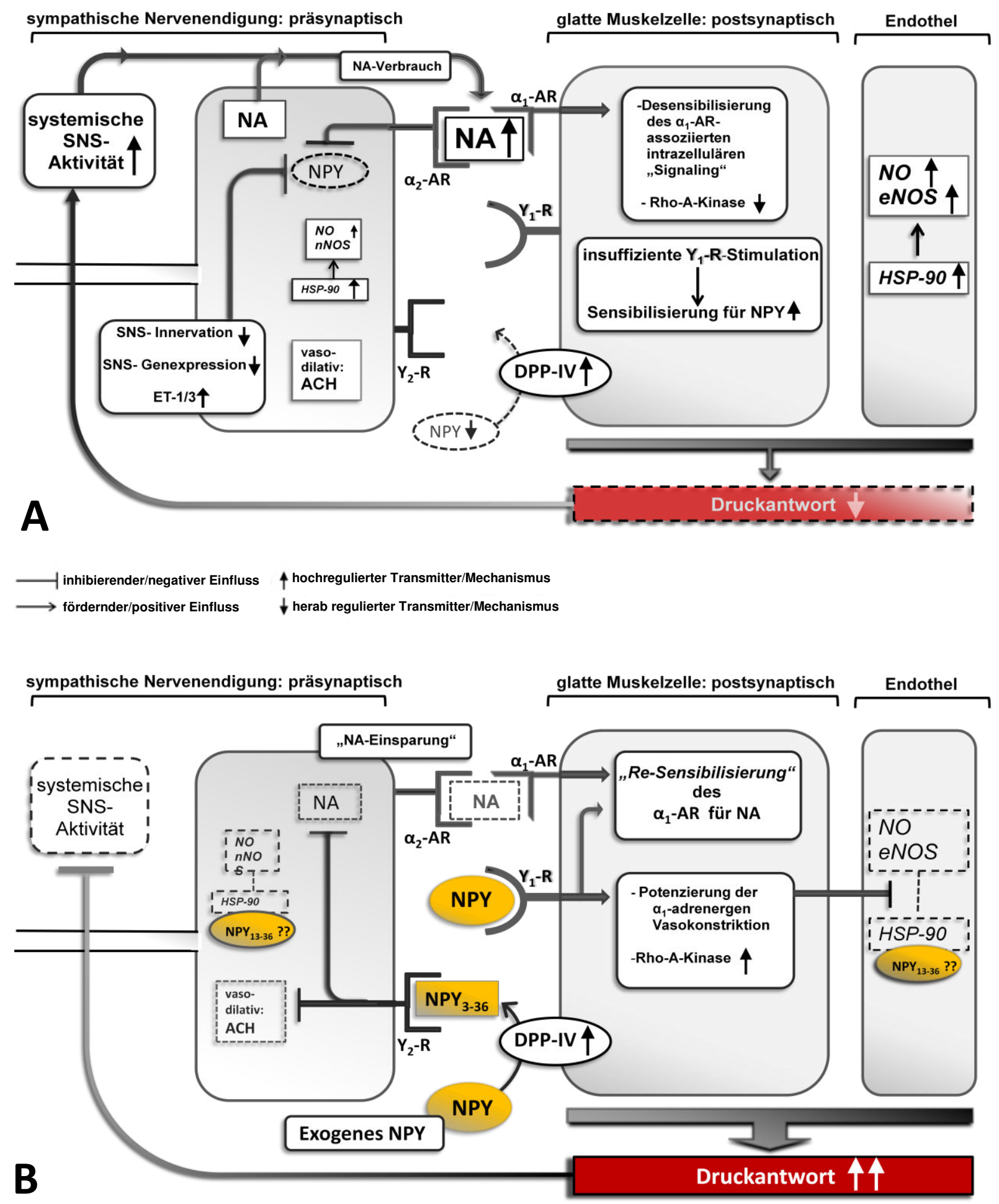

Abbildung 5: Hypothese der dysregulierten sympathischen Neurotransmitter-Sekretion im mesenterialen arteriellen Gefäßbett bei portaler Hypertension. A: Erhöhte NA-Freisetzung bei chronisch gesteigerter SNS-Aktivität führt im Bereich der glattmuskulären Signalübertragung zu prä- und postsynaptischen Effekten, welche zu einer verminderten vaskulären Druckantwort führen. B: Externes NPY kann die vasokonstriktive NA-Wirkung über den postsynaptischen $\mathrm{Y}_{1}$-Rezeptor und über präsynaptische $\mathrm{Y}_{1 / 2 / 5}$-Rezeptoren verstärken und ökonomisieren. Siehe Diskussion für eine detaillierte Erklärung. $\alpha_{1 / 2}-\mathrm{AR}$, $\alpha_{1 / 2}$-Adrenorezeptor. ACH, Acethylcholin. DPP-IV, Dipeptidyl-Peptidase-IV. ET-1/2, Endothelin-1/2. HSP-90, Hitze-SchockProtein-90. NO, Stickstoff-Monoxid. eNOS, endotheliale-NO-Synthase. nNOS, neuronale-NO-Synthase. SNS, sympathisches Nervensystem. $\mathrm{Y}_{1}-\mathrm{R}, \mathrm{Y}_{1}$-Rezeptor. 


\section{e. $\quad$ Referenzen}

[1] Wiest R and Groszmann RJ. The paradox of nitric oxide in cirrhosis and portal hypertension: Too much, not enough. Hepatology 2002;35:478-491.

[2] Hennenberg M, Trebicka J, Biecker E, Schepke M, Sauerbruch T, Heller J. Vascular dysfunction in human and rat cirrhosis: Role of receptor-desensitizing and calciumsensitizing proteins. Hepatology 2007;45:495-506.

[3] Llach J, Ginès P, Arroyo V, Rimola A, Titó L, Badalamenti S, et al. Prognostic value of arterial pressure, endogenous vasoactive systems, and renal function in cirrhotic patients admitted to the hospital for the treatment of ascites. Gastroenterology 1988;94(2):482-7.

[4] Braillon A, Gaudin C, Poo JL, Moreau R, Debaene B, Lebrec D. Plasma catecholamine concentrations are a reliable index of sympathetic vascular tone in patients with cirrhosis. Hepatology 1992;15(1):58-62.

[5] Gaudin C, Ruget G, Braillon A, Selz F, Cuche JL, Lebrec D. Portal and arterial free and conjugated noradrenaline in two models of portal hypertension in rats. Life Sci 1989;45(15):1333-9.

[6] Shaldon C, Peacock JH, Walker RM, Palmer DB, Badrick FE. The portal venous content of adrenaline and noradrenaline in portal hypertension. Lancet 1961;1(7184):957-61.

[7] Henriksen JH, Ring-Larsen H, Christensen NJ. Hepatic intestinal uptake and release of catecholamines in alcoholic cirrhosis. Evidence of enhanced hepatic intestinal sympathetic nervous activity. Gut 1987;28(12):163742.

[8] MacGilchrist A, Howes LG, Hawksby C, Reid JL. Plasma noradrenaline in cirrhosis: a study of kinetics and temporal relationship to ascites formation. Eur J Clin Invest 1991;21(2):238-43.

[9] Nicholls KM, Shapiro MD, Van Putten VJ, Kluge R, Chung HM, Bichet DG, et al. Elevated plasma norepinephrine concentrations in decompensated cirrhosis. Association with increased secretion rates, normal clearance rates, and suppressibility by central blood volume expansion. Circ Res 1985;56(3):457-461.

[10] Coll M, Martell M, Raurell I, Ezkurdia N, Cuenca S, Hernández-Losa J, et al. Atrophy of mesenteric sympathetic innervation may contribute to splanchnic vasodilation in rat portal hypertension. Liver Int 2010;30(4):593-602.

[11] Coll M, Genescà J, Raurell I, Rodríguez-Vilarrupla A, Mejías M, Otero T, et al. Downregulation of genes related to the adrenergic system may contribute to splanchnic vasodilation in rat portal hypertension. J Hepatol 2008;49(1):43-51.

[12] Ezkurdia N, Coll M, Raurell I, Rodriguez S, Cuenca S, González A. Blockage of the afferent sensitive pathway prevents sympathetic atrophy and hemodynamic alterations in rat portal hypertension. Liver Int 2012;32(8):1295-305

[13] Wahlestedt C, Edvinsson L, Ekblad E, Hakanson R. Neuropeptide Y potentiates noradrenaline evoked vasoconstriction: mode of action. J Pharmacol Exp Ther 1985;234(3):735-741.

[14] Cortés V, Donoso MV, Brown N, Fanjul R, López C, Fournier A, et al. Synergism between neuropeptide Y and norepinephrine highlights sympathetic cotransmission: studies in rat arterial mesenteric bed with neuropeptide Y, analogs, and BIBP 3226. J Pharmacol Exp Ther 1999;289(3):1313-22.

[15] Wiest R, Jurzik L, Moleda L, Froh M, Schnabl B, von Hörsten S, et al. Enhanced Y1-receptor-mediated vasoconstrictive action of neuropeptide Y (NPY) in superior mesenteric arteries in portal hypertension. J Hepatol 2006;44(3):512-9.

[16] Wiest R, Jurzik L, Herold T, Straub RH, Schölmerich J. Role of NPY for vasoregulation in the splanchnic circulation during portal hypertension. Peptides 2007;28(2):396-404. 
[17] Tsujimoto G, Honda K, Hoffman BB, Hashimoto K. Desensitization of postjunctional alpha 1- and alpha 2adrenergic receptor-mediated vasopressor responses in rat harboring pheochromocytoma. Circ Res 1987;61(1):8698 .

[18] Rosenbaum JS, Zera P, Umans VA, Ginsburg R, Hoffman BB. Desensitization of aortic smooth-muscle contraction in rats harboring pheochromocytoma. J Pharmacol Exp Ther 1986;238(2):396-400.

[19] Donoso MV, Brown N, Carrasco C, Cortes V, Fournier A, Huidobro-Toro JP. Stimulation of the sympathetic perimesenteric arterial nerves releases neuropeptide $\mathrm{Y}$ potentiating the vasomotor activity of noradrenaline: involvement of neuropeptide Y-Y1 receptors. J Neurochem 1997;69(3):1048- 59.

[20] Han S, Yang CL, Chen X, Naes L, Cox BF, Westfall T. Direct evidence for the role of neuropeptide Y in sympathetic nerve-stimulation-induced vasoconstriction. Am J Physiol 1998;274(1 Pt 2):H290-4.

[21] McGregor DD. The effect of sympathetic nerve-stimulation on vasoconstrictor responses in perfused mesenteric vessels of the rat. J Physiol 1965;177:21-30.

[22] Sikuler E, Kravetz D, Groszmann RJ. Evolution of portal hypertension and mechanisms involved in its maintenance in a rat model. Am J Physiol 1985;248(6 Pt 1):G618-25.

[23] Capellino S, Cosentino M, Wolff C, Schmidt M, Grifka J, Straub RH. Catecholamine-producing cells in the synovial tissue during arthritis: modulation of sympathetic neurotransmitters as new therapeutic target. Ann Rheum Dis 2010;69(10):1853-60.

[24] Rosas-Ballina M, Olofsson PS, Ochani M, Valdés-Ferrer SI, Levine YA, Reardon C, et al. Acetylcholinesynthesizing T cells relay neural signals in a vagus nerve circuit. Science 2011 Oct;334(6052):98-101.

[25] Straub RH, Grum F, Strauch U, Capellino S, Bataille F, Bleich A, et al. Anti-inflammatory role of sympathetic nerves in chronic intestinal inflammation. Gut 2008;57(7):911-21.

[26] Capellino S, Weber K, Gelder M, Härle P, Straub RH. First appearance and location of catecholaminergic cells during experimental arthritis and elimination by chemical sympathectomy. Arthritis Rheum 2012;64(4):11108.

[27] Haass M, Cheng B, Richardt G, Lang RE, Schömig A. Characterization and presynaptic modulation of stimulation-evoked exocytotic co-release of noradrenaline and neuropeptide $\mathrm{Y}$ in guinea pig heart. Naunyn Schmiedebergs Arch Pharmacol 1989;339(1-2):71-8.

[28] Pernow J, Lundberg JM. Modulation of noradrenaline and neuropeptide Y (NPY) release in the pig kidney in vivo: involvement of alpha-2, NPY and angiotensin II receptors. Naunyn Schmiedebergs Arch Pharmacol 1989;340(4):379-85.

[29] Hall GT, Gardner TD, Potter EK. Attenuation of long-lasting effects of sympathetic stimulation after repeated stimulation. Circ Res 1990;67(1):193-8.

[30] Lakatos PL, Firneisz G, Rákóczy G, Selmeci L, Szalay F. Elevated serum dipeptidyl peptidase IV (CD26, EC 3.4.14.5) activity in patients with primary biliary cirrhosis. J Hepatol 1999;30(4):740.

[31] Palmieri FE, Ward PE. Dipeptidyl(amino)peptidase IV and post proline cleaving enzyme in cultured endothelial and smooth-muscle cells. Adv Exp Med Biol 1989;247A:305-11.

[32] Mentlein R, Dahms P, Grandt D, Krüger R. Proteolytic processing of neuropeptide Y and peptide YY by dipeptidyl peptidase IV. Regul Pept 1993;49(2):133-44.

[33] Hoang D, Macarthur H, Gardner A, Westfall TC. Endothelin-induced modulation of neuropeptide Y and norepinephrine release from the rat mesenteric bed. Am J Physiol Heart Circ Physiol 2002;283(4):H1523-30.

[34] Asbert M, Ginès A, Ginès P, Jiménez W, Clària J, Saló J, et al. Circulating levels of endothelin in cirrhosis. Gastroenterology 1993;104(5):1485-91. 
[35] Bernardi M, Gulberg V, Colantoni A, Trevisani F, Gasbarrini A, Gerbes AL. Plasma endothelin-1 and -3 in cirrhosis: relationship with systemic hemodynamics, renal function and neurohumoral systems. J Hepatol $1996 ; 24(2): 161-8$.

[36] Wiest R, Moleda L, Zietz B, Hellerbrand C, Schölmerich J, Straub R. Uncoupling of sympathetic nervous system and hypothalamic-pituitary-adrenal axis in cirrhosis. J Gastroenterol Hepatol 2008;23(12):1901-8.

[37] Liao JF, Yu PC, Lin HC, Lee FY, Kuo JS, Yang MC. Study on the vascular reactivity and alpha 1adrenoceptors of portal hypertensive rats. Br J Pharmacol 1994;111(2):439-44.

[38] Moleda L, Trebicka J, Dietrich P, Gäbele E, Hellerbrand C, Straub RH, et al. Amelioration of portal hypertension and the hyperdynamic circulatory syndrome in cirrhotic rats by neuropeptide $\mathrm{Y}$ via pronounced splanchnic vasoaction. Gut 2011;60(8):1122-32.

[39] Westfall TC, Naes L, Gardner A, Yang CL. Neuropeptide Y induced attenuation of catecholamine synthesis in the rat mesenteric arterial bed. J Cardiovasc Pharmacol 2006;47(6):723-8.

[40] Jurzik L, Froh M, Straub RH, Schölmerich J, Wiest R. Up-regulation of nNOS and associated increase in nitrergic vasodilation in superior mesenteric arteries in pre-hepatic portal hypertension. J Hepatol 2005;43(2):25865 .

[41] Wiest R, Shah V, Sessa WC, Groszmann RJ. NO overproduction by eNOS precedes hyperdynamic splanchnic circulation in portal hypertensive rats. Am J Physiol 1999;276(4 Pt 1):G1043-51.

[42] Kolo LL, Westfall TC, Macarthur H. Nitric oxide decreases the biological activity of norepinephrine resulting in altered vascular tone in the rat mesenteric arterial bed. Am J Physiol Heart Circ Physiol 2004;286(1):H296-303.

[43] Moleda L, Jurzik L, Froh M, Gäbele E, Hellerbrand C, Straub RH, et al. Role of HSP-90 for increased nNOSmediated vasodilation in mesenteric arteries in portal hypertension. World J Gastroenterol 2010;16(15):1837-44.

[44] Shah V, Wiest R, Garcia-Cardena G, Cadelina G, Groszmann RJ, Sessa WC. Hsp90 regulation of endothelial nitric oxide synthase contributes to vascular control in portal hypertension. Am J Physiol 1999;277(2 Pt 1):G463-8.

[45] Ishiwatari-Hayasaka H, Maruya M, Sreedhar AS, Nemoto TK, Csermely P, Yahara I. Interaction of neuropeptide Y and Hsp90 through a novel peptide binding region. Biochemistry 2003;42(44):12972-80.

[46] Hendriks MG, Pfaffendorf M, Van Zwieten PA. Characterization of the muscarinic receptor subtype mediating vasodilation in the rat perfused mesenteric vascular bed preparation. $J$ Auton Pharmacol $1992 ; 12(6): 411-20$

[47] Mahns DA, Revington ML, Runcie MJ, McCloskey DI, Potter EK. Inhibition of sympathetic cholinergic vasodilatation by a selective NPY Y2 receptor agonist in the gracilis muscle of anaesthetised dogs. J Auton Nerv Syst 1998;68(1-2):14-20.

[48] Ahlborg G, Lundberg JM. Splanchnic release of neuropeptide Y during prolonged exercise with and without beta-adrenoceptor blockade in healthy man. Clin Physiol 1991;11(4):343-51.

[49] Ahlborg G, Weitzberg E, Sollevi A, Lundberg JM. Splanchnic and renal vasoconstrictor and metabolic responses to neuropeptide Y in resting and exercising man. Acta Physiol Scand 1992;145(2):139-49.

[50] Mercer RE, Chee MJ, Colmers WF. The role of NPY in hypothalamic mediated food intake. Front Neuroendocrinol 2011;32(4):398-415. 


\title{
Dysbalance in sympathetic neurotransmitter release and action in cirrhotic rats: Impact of exogenous neuropeptide $Y$
}

\author{
P. Dietrich ${ }^{1}$, L. Moleda ${ }^{1}$, F. Kees ${ }^{2}$, M. Müller ${ }^{1}$, R.H. Straub ${ }^{1}$, C. Hellerbrand ${ }^{1}$, R. Wiest ${ }^{3, *}$ \\ ${ }^{1}$ Dept. Internal Medicine, University Medical Center, Regensburg 93042, Germany; ${ }^{2}$ Dept. Pharmacology and Toxicology, University of \\ Regensburg, Regensburg 93040, Germany; ${ }^{3}$ Dept. Visceral Surgery and Medicine, Gastroenterology, University Medical Center,
} Bern 3010, Switzerland

\begin{abstract}
Background \& Aims: Splanchnic vasodilation is an essential disturbance in portal hypertension. Increased systemic sympathetic nerve activity is well known, but potential corresponding vascular desensitization is incompletely characterized. Release of splanchnic sympathetic neurotransmitters noradrenaline (NA) and co-transmitter neuropeptide Y (NPY) remains to be elucidated. Finally, the effects of exogenous NPY on these mechanisms are unexplored.

Methods: Portal vein ligated cirrhotic, and control rats were used for in vitro perfusion of mesenteric arteries. Depletion of vascular pressure response was induced by repetitive electric sympathetic perivascular nerve stimulation (PNS) and performed in the absence and presence of exogenous NPY. Additionally, PNSinduced release of NA and NPY was measured.

Results: Mesenteric PNS-induced pressure response was lower in portal hypertension. Depletion of the pressure response to PNS, representing the degree of desensitization, was enhanced in portal hypertension. NA release was elevated, whereas NPY release was attenuated in cirrhosis. Administration of exogenous NPY led to marked recovery from desensitization and vasoconstrictive improvement in cirrhotic rats, being associated with more pronounced decrease of NA release.

Conclusions: Pronounced depletion of splanchnic arterial pressure-response to repetitive sympathetic nerve stimulation in cirrhosis is partly attributable to altered NA release as well as to deficient NPY release. External NPY restores vascular contractility and attenuates pathologically elevated NA release in the portal hypertensive mesenteric vasculature, revealing post-, and prejunctional effects at the vascular smooth muscle motor endplate; therefore outlining encouraging therapeutic strategies.
\end{abstract}

Keywords: Neuropeptide Y; Noradrenaline; Perivascular nerve-stimulation; Portal hypertension; Splanchnic circulation.

Received 30 April 2012; received in revised form 24 September 2012; accepted 25 September 2012; available onilne 4 October 2012

* Corresponding author. Tel.: +41 31632 0291; fax: +41 316322988.

E-mail address: reiner.wiest@insel.ch (R. Wiest).

Abbreviations: ACH, acethylcholine; b.w., bodyweight; DPP-IV, dipeptidyl-peptidase-IV; ET-1/2, endothelin-1/2; HSP-90, heat-shock-protein-90; LC, liver cirrhosis; NA, noradrenaline (norepinephrine); NPY, neuropeptide Y; eNOS, endothelialnitric-oxide-synthase; nNOS, neuronal-nitric-oxide-synthase; NO, nitric oxide PNS, electric perivascular-nerve-stimulation; PR, pressure-response; PVL, portal vein ligation; $r$, pearson correlation coefficient (pcc); $\mathrm{R}^{2}$, coefficient of determination; SE, standard error; SMA, superior mesenteric artery; SNS, sympatheticnervous-system. (c) 2012 European Association for the Study of the Liver. Published by Elsevier B.V. All rights reserved.

\section{Introduction}

Splanchnic arterial vasodilation in portal hypertension is attributed to overproduction of vasodilators and hyporeactivity to vasoconstrictors [1], which is mediated partly by defective intracellular vascular smooth muscle cell signaling [2]. Upregulated sympathetic nervous system (SNS), acting as compensatory mechanism, reveals prognostic significance, namely increased serum levels of noradrenaline (NA), correlating with survival of cirrhotic patients $[3,4]$. In case of portal hypertension, systemic plasma NA levels could mainly depend on splanchnic NA production [5]. Besides systemic NA levels, data in terms of actual splanchnic synthesis/release of neurotransmitters are scarce and controversial [6-12].

Neuropeptide Y (NPY) is co-stored and co-released with NA from secretory vesicles of sympathetic nerve terminals, inducing potentiation of $\alpha_{1}$-adrenergic vasoconstriction via G-proteinassociated postsynaptic $Y_{1}$ receptors [13]. It mediates this effect via sensitizing vascular smooth muscle to NA, although the exact pathway is unknown [14]. We previously found that NPY augments $\alpha_{1}$-adrenergic mesenteric vasoconstriction induced by exogenous NA in cirrhotic rats to a greater extent than in healthy animals $[15,16]$.

Prolonged adrenergic tissue stimulation leads to diminished responsiveness to subsequent activation by catecholamines; this general process is termed "desensitization". Consequently, sustained SNS activity, as seen in pheochromocytoma, may lead to vascular desensitization, thereby aggravating splanchnic vasodilation in cirrhosis as well $[17,18]$.

In the isolated rat mesenteric bed, electric sympathetic nerve stimulation mimics extensive and/or prolonged SNS activity. This procedure has been examined previously in healthy rats and causes pressure response by inducing NA and NPY release $[19,20]$. However, the physiological stimulus for the sympathoadrenergic modulation of vascular tone, namely nerve stimulation, has not been used so far for direct evaluation of SNS neurotransmitter release and associated hemodynamic action in portal hypertensive splanchnic vasculature. Therefore, we aimed firstly at exploring the hemodynamic effect of mesenteric vascular 
desensitization caused by repetitive perivascular nerve stimulation with, secondly, concomitant assessment of PNS-induced SNS neurotransmitter release in portal hypertensive rats. Thirdly, the impact of exogenous NPY on PNS-induced pressure response and SNS neurotransmitter release was addressed.

\section{Materials and methods}

Animals

Experiments were conducted according to the German Physiological Society principles for the use of laboratory animals (Granted permission number 621-2531.123/00, Government of Bavaria).

$\mathrm{CCl}_{4}$-induced liver cirrhosis (LC)

Cirrhosis was induced in male specific pathogen-free rats (Charles-River) by $\mathrm{CCl}_{4}$ inhalation along with phenobarbital $(0.35 \mathrm{~g} / \mathrm{L})$ containing drinking water. After 12-16 weeks, this approach induces cirrhosis. Age- and sex-matched rats were used as control group (Con).

Induction of portal hypertension: Portal vein ligation (PVL)

A prehepatic portal hypertensive animal model extensively studied in our laboratory was used $[15,16]$. Briefly, after a midline abdominal incision, the portal vein was freed from the surrounding tissue. A ligature was placed around a needle lying alongside the portal vein. Subsequent removal of the needle yielded a calibrated stenosis.

In vitro perfusion

The in vitro perfusion system used was a modification of that originally described by McGregor and used extensively in our laboratory [21]. After isolating the superior mesenteric artery (SMA) with its mesentery, splanchnic vasculature was perfused with oxygenated $37^{\circ} \mathrm{C}$-Krebs solution using a roller pump (Isamtec, IPC-8channel; Glattburg, Zürich, Switzerland). Perfusion pressure was measured with a P-23-Db strain gauge transducer (Statham) and continuously recorded (Powerlab Quadbridge and Powerlab 4/20; AD Instruments).

Perivascular nerve stimulation (PNS)

Nerves surrounding the SMA were stimulated $(33 \mathrm{~Hz}, 50 \mathrm{~V}, 30 \mathrm{~s})$ using a nerve stimulator (I-ZQ4V, Hugo Sachs Electronics). In preliminary experiments, we investigated the time and frequency dependent induction of the vascular pressure response (PR). We confirm that PNS at $33 \mathrm{~Hz}$ and 30 s causes maximal PR, which cannot be increased further by higher frequencies and/or longer stimulation times [19]. PNS provokes reproducible peaks of PR by stimulating endogenous vasoconstrictor release [19]

Quantification of noradrenaline (NA)

Aliquots of 3-ml perfusate were collected (collection tubes containing $60 \mu \mathrm{l} \mathrm{GSH}$ / EGTA solution as preservative). A Prominence HPLC system (Shimadzu, Duisburg, Germany) with an amperometric detector $3500 \mathrm{~A}$ and Sputnik ${ }^{\circledR}$ detector cell (Recipe, Munich/Germany) was used. Aliquots of 1-1.5 ml perfusate were analysed with a commercially available kit (ClinRep ${ }^{\circledR}$ Catecholamines in plasma, Recipe, Munich/Germany). As low as $5 \mathrm{pg} / \mathrm{ml}$ NA could be determined.

Quantification of neuropeptide Y (NPY)

A commercial radioimmunoassay kit by Eurodiagnostica (EURIA-NPY) was used. Crossreaction with other peptides was below $0.1 \%$. The lowest detectable concentration was $3 \mathrm{pmol} / \mathrm{L}$. The mean recovery range was $75-88 \%$.

Protocol I: desensitization to repetitive PNS and neurotransmitter release

This protocol was performed to characterize the extent of pressure response degradation caused by repetitive SNS neurotransmitter release [Con: $n=19$, PVL: $\mathrm{n}=12$, LC: $\mathrm{n}=19$ ]. After baseline perfusion at $4 \mathrm{ml} / \mathrm{min}$ was established for

\section{JOURNAL OF HEPATOLOGY}

$30 \mathrm{~min}, 15$ repetitive PNS were implemented (interval-time: $8 \mathrm{~min}$ ). This first period of repetitive PNS is called the 1st perfusion cycle. To compare endogenous NA and NPY release, perfusate samples were collected after 3rd, 6th, 9th, 12th, and 15th PNS in another series of experiments [Con: $n=12$, LC: $n=12$ ]. In PVL rats, hyperdynamic-circulation develops acutely (within 3-5 days) [22]. Portal hypertension decreases afterwards due to the development of portosystemic collaterals, whereas compensatory SNS upregulation and altered NA release in cirrhosis emerge chronically [22]. In cirrhotic rats, systemic NA levels are higher than in PVL rats, indicating that enhanced sympathetic nerve activity depends on the development of cirrhosis [5]. Therefore, PVL rats were not used for NA measurement.

Protocol II: prolonged effects of pressure response (PR) depletion and impact of exogenous NPY

After the 1st perfusion cycle, a 1-h washout period was performed, followed by a 2nd perfusion cycle with 5 sequential PNS (PNS-1b-5b) to investigate the sustained effects of PR depletion. To characterize the influence of exogenous NPY, the groups were divided [Con: $n=8$, Con + NPY: $n=8$, PVL: $n=6, P V L+N P Y$ : $n=6$, LC: $n=11$, LC + NPY: $n=11]$. In NPY groups, exogenous NPY [ $50 n M]$ was added to the perfusion solution halfway through the 1-h washout period. In preliminary studies, we found $50 \mathrm{nM}$ NPY having no direct vasoconstrictive action. NPY was present at the same molar concentration in the perfusion system throughout the 2nd perfusion cycle. For NA measurement, samples were collected similar to protocol $I$ in another series of experiments [Con: $n=12$, LC: $\mathrm{n}=12$ ]. In the 2nd perfusion cycle, NPY release was not measured, because interacting exogenous NPY would hinder interpretation.

Statistical analysis

Normal distribution was tested by Kolmogorov-Smirnov-test. Values were compared using ANOVA (one-way, with repeated measurements and correction for multiple comparisons) or the Student's- $t$ test if appropriate. The significance level was set at $p<0.05$

\section{Results}

Animals

Spleen weight was elevated in portal hypertension (Table 1). Pressure response to PNS correlated negatively and significantly with spleen weight $(r=-0.86, p<0.01)$ being apparently logarith$\operatorname{mic}\left(R^{2}=0.8\right)$.

\section{Repetitive PNS and pressure response (PR) [1st perfusion cycle]}

Baseline perfusion pressure was lower in portal hypertensive rats (Table 1). PR was maximal at PNS-3 and decreased with each further PNS, reflecting a desensitization process. PR was blunted in portal hypertension (Fig. 1A). The decline in pressure response was estimated relative to maximum (PNS-3). This process was pronounced in portal hypertensive study groups, indicating an accelerated decline of pressure response to repetitive PNS (Fig. 1B). In fact, in portal hypertensive mesenteric arteries, significantly less numbers of PNS were necessary to achieve, e.g., a 40\%-reduction (Table 1) from maximum pressure response.

\section{PNS-induced NA release and associated PR [1st perfusion cycle]}

Basal NA release was low in both study groups (Con: $13.6 \pm 9.6 \mathrm{pg} / \mathrm{ml} v$ s. LC: $15.0 \pm 6.2 \mathrm{pg} / \mathrm{ml}$; n.s.). PNS-induced NA release was higher in cirrhosis (Fig. 1C). With increasing numbers of PNS, NA release degraded . The slope of decrease and the overall decrease of NA release were more pronounced in cirrhotic rats $(477.5 \pm 121.6 \mathrm{pg} / \mathrm{ml})$ as compared to controls $(219.9 \pm 110.9$ 


\section{Research Article}

Table 1. Basic and haemodynamic parameters [1st perfusion cycle].

\begin{tabular}{|c|c|c|c|}
\hline Parameter & Con & PVL & LC \\
\hline Spleen-weight/body weight [g/kg b.w.] & $1.7 \pm 0.3$ & $4.2 \pm 1.0^{\star * \star}$ & $6.0 \pm 0.9^{* \star *}$ \\
\hline Spleen-weight/mean PR in 1st PC [g/mmHg] & $0.005 \pm 0.001$ & $0.019 \pm 0.005^{\star \star \star}$ & $0.044 \pm 0.014^{\star \star *}$ \\
\hline Baseline [mmHg] & $21.4 \pm 6.3$ & $15.4 \pm 3.3^{*}$ & $16.3 \pm 4.1^{*}$ \\
\hline Mean pressure-response $=\mathrm{PR}[\mathrm{mmHg}]$ & $114.3 \pm 17.5$ & $74 \pm 9.5^{* * *}$ & $57.4 \pm 11.3^{\star * *}$ \\
\hline Decrease of PR [\%] & $34.6 \pm 17.1$ & $55.1 \pm 10.9^{\star * *}$ & $50.4 \pm 8.1^{* * *}$ \\
\hline DP-40 & $12.8 \pm 2.3$ & $9.8 \pm 2.8^{\star *}$ & $10.2 \pm 3^{* *}$ \\
\hline
\end{tabular}

DP-40, number of PNS inducing a 40\%-decrease of pressure response.

${ }^{*} p<0.05$ vs. Con; ${ }^{* *} p<0.01$ vs. Con; ${ }^{* * *} p<0.001$ vs. Con.

A

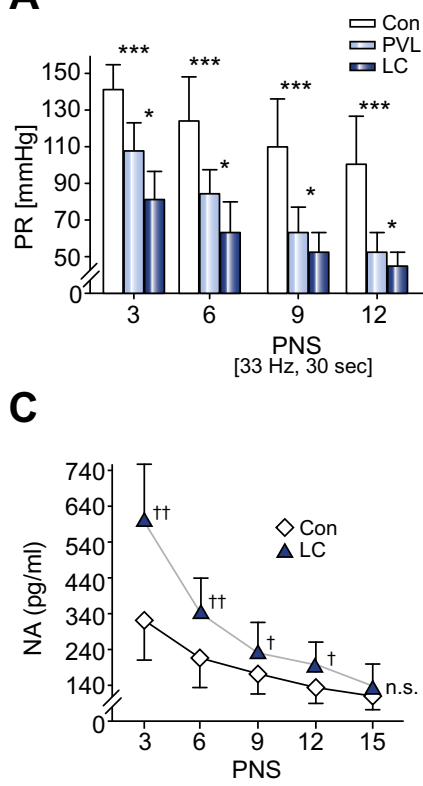

B

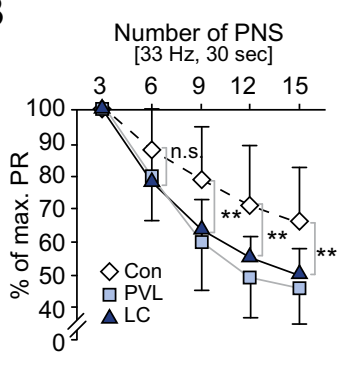

D

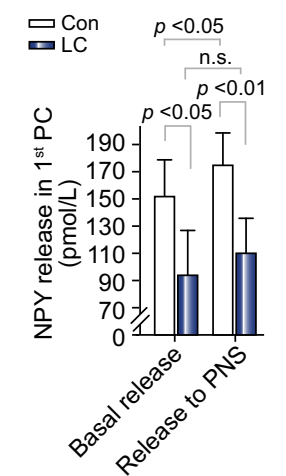

Fig. 1. Pressure response (PR) and neurotransmitter release [1st perfusion cycle]. (A) Lower PNS-induced PR [PVL: $n=12$, LC: $n=19$, Con: $n=19]$ and (B) pronounced PR depletion in PVL and LC. (C) NA release is higher in LC $(n=12)$ as compared to controls $(n=12)$. (D) Basal and PNS-induced NPY release is reduced in LC. ${ }^{*} p<0.05$ vs. LC; ${ }^{* *} p<0.01$ vs. LC/PVL; ${ }^{* * *} p<0.001$ vs. other groups; ${ }^{\dagger} p<0.05$ and ${ }^{\dagger \dagger} p<0.01$ vs. Con; n.s., not significant. LC, liver cirrhosis; PC, perfusion cycle; PNS, periarterial nerve stimulation; PVL, portal vein ligation.

$\mathrm{pg} / \mathrm{ml} ; p<0.001)$. In order to estimate the efficacy of NA in causing vasoconstriction, we calculated the ratio NA release/PR. The more NA is necessary to change pressure response by, e.g., $1 \mathrm{mmHg}$, the higher this ratio is. In fact, cirrhotic rats did exhibit higher NA/PR ratios (LC: $5.7 \pm 2.2[\mathrm{pg} / \mathrm{ml}] / \mathrm{mmHg}$ vs. Con: $1.7 \pm 0.5[\mathrm{pg} / \mathrm{ml}] / \mathrm{mmHg} ; p<0.01)$.

\section{PNS-induced NPY release [1st perfusion cycle]}

Basal NPY release as well as PNS-induced NPY release from mesenteric vasculature was lower in cirrhosis (Fig. 1D). In contrast to the decrease of PNS-induced NA release, NPY release in both study groups showed no abatement (data not shown): the amount of PNS-induced NPY release was independent of the

number and/or intensity of nerve stimulation. Compared to basal NPY release, PNS induced a significant increase of NPY release only in healthy rats (Fig. 1D). Consequently, the NPY/NA ratio was lower in cirrhosis $(0.6 \pm 0.2[\mathrm{pmol} / \mathrm{L}] /[\mathrm{pg} / \mathrm{ml}])$ as compared to controls $(1.2 \pm 0.3[\mathrm{pmol} / \mathrm{L}] /[\mathrm{pg} / \mathrm{ml}] ; p<0.001)$, also underlining a deficiency in NPY release.

Pressure response and impact of exogenous NPY [2nd perfusion cycle]

Like in the 1st perfusion cycle, basal perfusion pressure before 2nd perfusion cycle was lower in LC and PVL rats $(p<0.05)$, and not altered by incubation with NPY (data not shown). PNSinduced pressure response was blunted in portal hypertension (Fig. 2B). PNS-induced PR depletion along the 2 nd perfusion cycle was significant only in portal hypertensive rats and flattened in the presence of NPY (Fig. 2A). NPY slightly improved pressure response in controls but markedly improved pressure response in PVL and LC rats (Fig. 2B). To understand the extent of time dependent recovery from PR depletion caused by repetitive PNS in the 1 st perfusion cycle, we compared pressure response to the first PNS of the 2nd perfusion cycle [PNS-1b] to pressure response to the last PNS in the 1st perfusion cycle [PNS-15]. Thus, the ratio [PNS-1b/PNS-15] was used as indicator for recovery of vascular ability in causing pressure response after the 1-h washout period (Fig. 3A). Without NPY, after $1 \mathrm{~h}$, no recovery of pressure response could be seen in any group (Fig. $3 \mathrm{C}-\mathrm{E}$ ). In controls, exogenous NPY could not significantly improve the ratio [PNS-1b/ PNS-15] (Fig. 3C). In portal hypertensive rats, however, NPY significantly elevated this ratio, being able to markedly restore pressure response (Fig. 3B, D, and E). Indeed, NPY caused a $32.5 \pm 50.1 \%$ increase in vascular sensitivity to PNS in healthy rats (n.s.) vs. e.g., $114.3 \pm 35.5 \%$ enhancement of pressure response in LC rats $(p<0.001)$.

\section{NA release and impact of exogenous NPY [2nd perfusion cycle]}

Like in the 1st perfusion cycle, LC rats showed elevated PNSinduced NA release. Exogenous NPY did slightly blunt NA release in controls. In LC rats, PNS-induced NA release was drastically suppressed in presence of exogenous NPY (Fig. 2C). Like in the 1 st perfusion cycle, cirrhotic rats showed again more than threefold elevated NA/PR ratios. In the presence of exogenous NPY, the pathologically increased NA/PR ratios in cirrhosis were markedly reduced to levels equal to those of controls (Fig. 2D). 


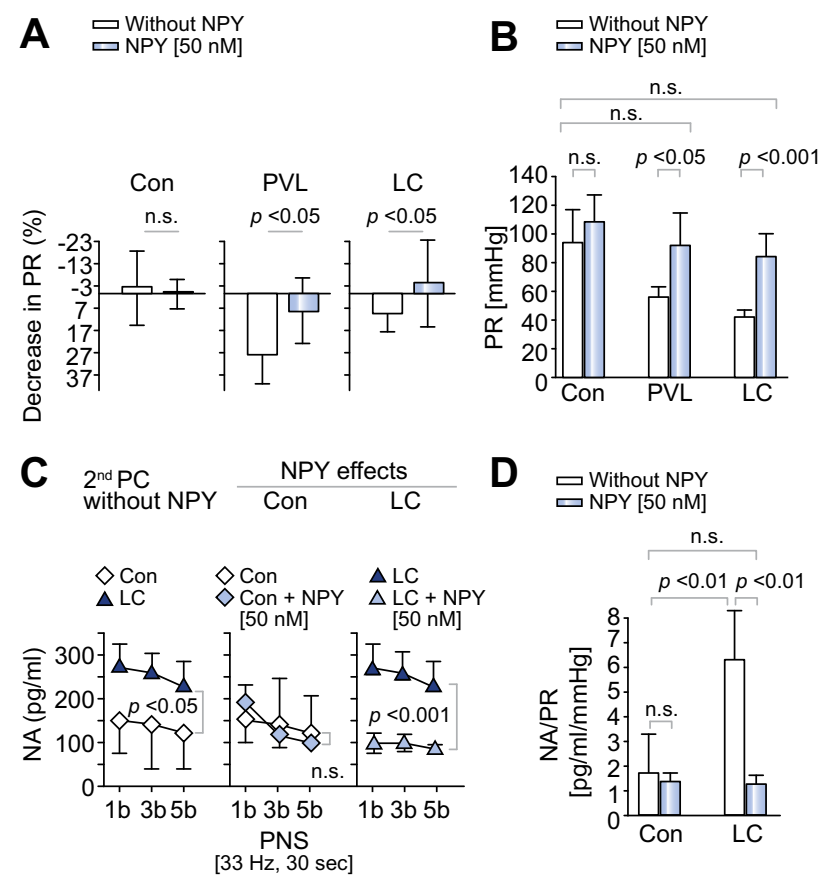

Fig. 2. Pressure-response, NA release and impact of exogenous NPY in the 2nd perfusion cycle. Impact of exogenous NPY [50 nM] on (B) PNS-induced pressure response and (A) PR depletion. Con: $n=8$, Con + NPY: $n=8$, PVL: $n=6$, PVL + NPY: $n=6, L C: n=11, L C+N P Y: n=11$. (C) PNS-induced NA release and impact of exogenous NPY [Con: $n=6$, Con + NPY: $n=6$, LC: $n=6$, LC + NPY: $n=6$ ]. (D) The ratio NA release/PR functions as index of NA efficacy in causing PR and is elevated in LC, this imbalance is compensated by exogenous NPY. n.s., not significant. LC liver cirrhosis; PNS, periarterial nerve stimulation; PR, pressure response; PVL, portal vein ligation.

\section{Discussion}

Numerous investigations underline marked SNS activity in cirrhosis [3,6-9]. However, recent reports suggest splanchnic sympathetic atrophy in portal hypertension. In detail, marked reduction of sympathetic nerves and decreased protein expression of tyrosine-hydroxylase key enzyme for NA synthesis have been demonstrated in portal hypertensive mesenteric arteries [10-12]. Applying a model of repetitive electric PNS resembling sustained splanchnic vascular SNS activity, we report that cirrhotic rats display almost doubled and about 30\% increased NA release caused by first and subsequent PNS, respectively. This apparent discrepancy can potentially be explained by peripheral immune cells being equipped with catecholamine synthesizing enzymes [23]. Indirect evidence also shows neurotransmitter release from immune cells in response to electrical stimulation [24]. In fact, in face of the loss of sympathetic nerve fibers, which is a feature of many chronic inflammatory diseases [25], concomitant increase in tyrosine-hydroxylase positive immune cells has been demonstrated [26]. Considering the well-known splanchnic chronic low level inflammation in ascitic cirrhosis, it is tempting to speculate whether an enhanced infiltration with catecholaminergic immune cells may contribute to the increased PNSinduced NA release. However, this was not the aim of the current investigation and has to be addressed by further studies. Nonetheless, our data concur with previous indirect evidence of enhanced splanchnic NA release showing increased NA concentrations in portal venous as compared to arterial and/or peripheral blood samples in human [6] and experimental cirrhosis [5].

\section{JOURNAL OF HEPATOLOGY}
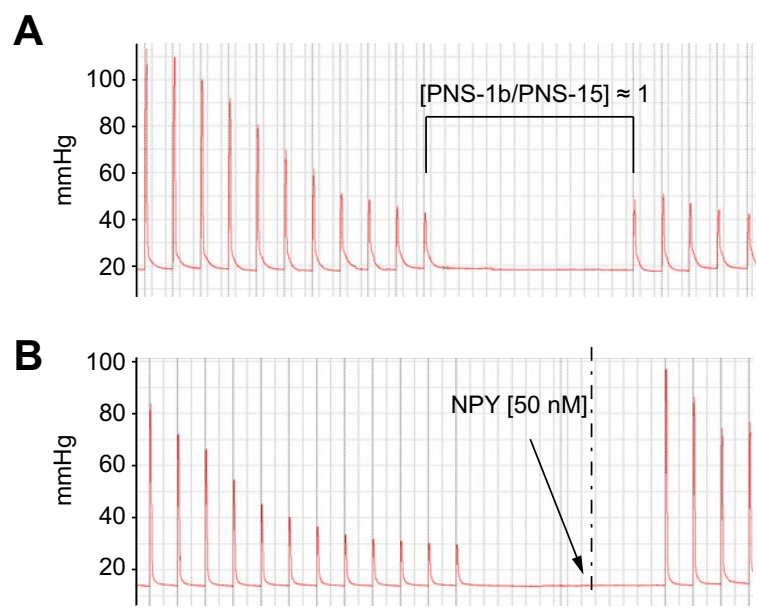

C

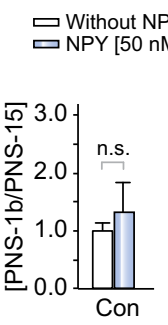

D $\square$ Without NPY
$\square$ NPY [50 nM]

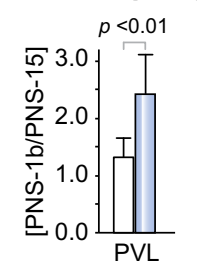

E Without NPY
NPY [50 nM]

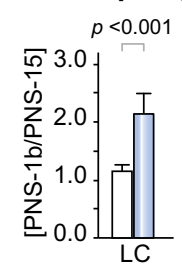

Fig. 3. Recovery of pressure response in presence/absence of exogenous NPY (A) A representative chart displays repetitive PNS [1st perfusion cycle] followed by a 1 -h washout period and a 2 nd perfusion cycle including 5 repetitive PNS. (B) Recovery of PR to PNS of an LC rat in presence of exogenous NPY. NPY significantly improves PR, given as ratio [PR to PNS-1b]/[PR to PNS-15], in (E) LC and (D) PVL, but not (C) in controls. Con: $n=8$; Con + NPY: $n=8$; PVL: $n=6$; PVL + NPY: $n=6 ; \quad L C: n=11 ; \quad L C+N P Y: n=11$. n.s., not significant. LC, liver cirrhosis; PC, perfusion cycle; PNS, periarterial nerve stimulation; PR, pressure response; PVL, portal vein ligation. (This figure appears in color on the web.)

In contrast, splanchnic PNS-induced NPY release was blunted in cirrhosis. Significant increases in PNS-induced NPY release were detected only in healthy rats, demonstrating a diminished ability of sympathetic nerves to release NPY in portal hypertensive splanchnic arteries. This could represent the functional correlate for reported downregulation and regression of sympathetic innervation in the mesenteric arterial tree in cirrhosis $[10,11]$. Besides local SNS downregulation, other potential mechanisms could explain NPY deficiency: unquestionably, systemic NA levels are increased in cirrhosis [3,6-9]. This might lead to enhanced $\alpha_{2}$-adrenergic presynaptic inhibition of NPY release $[27,28]$. Indeed, repeated stimulation of sympathetic nerves causes depletion of NPY release [29]. Another interesting linkage to decreased splanchnic NPY action is elevated dipeptidyl-peptidase IV (DPP-IV) activity in cirrhosis [30]. DPP-IV acts on the extracellular metabolism of peptides at/near endothelial and smooth muscle cell surface receptors [31]. Proteolytic processing of NPY by DPP-IV is well known [32]. Consequently elevated DPPIV activity in cirrhosis might lead to enhanced NPY processing and thus reduced mesenteric NPY activity. Furthermore, dysbalanced SNS neurotransmitter release is in accordance with evidence of differential modulation of NA and NPY release in the rat mesenteric bed: endothelins (ET-1, ET-3) have been shown to inhibit NPY release but not NA release [33], closely resembling our results. Considering cirrhosis, circulating plasma levels of 


\section{Research Article}

\section{A}

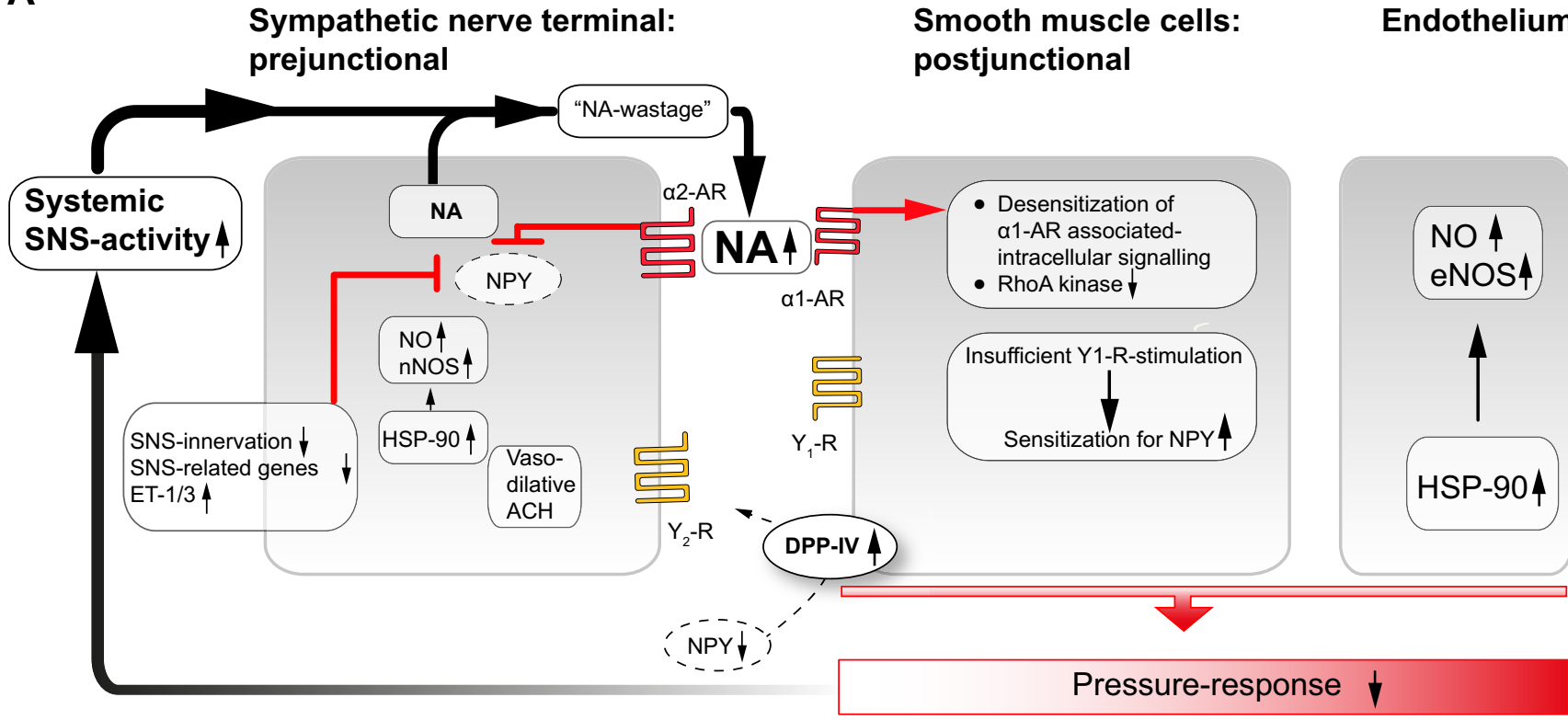

B

\section{Sympathetic nerve terminal: prejunctional}

\section{Smooth muscle cells: postjunctional}

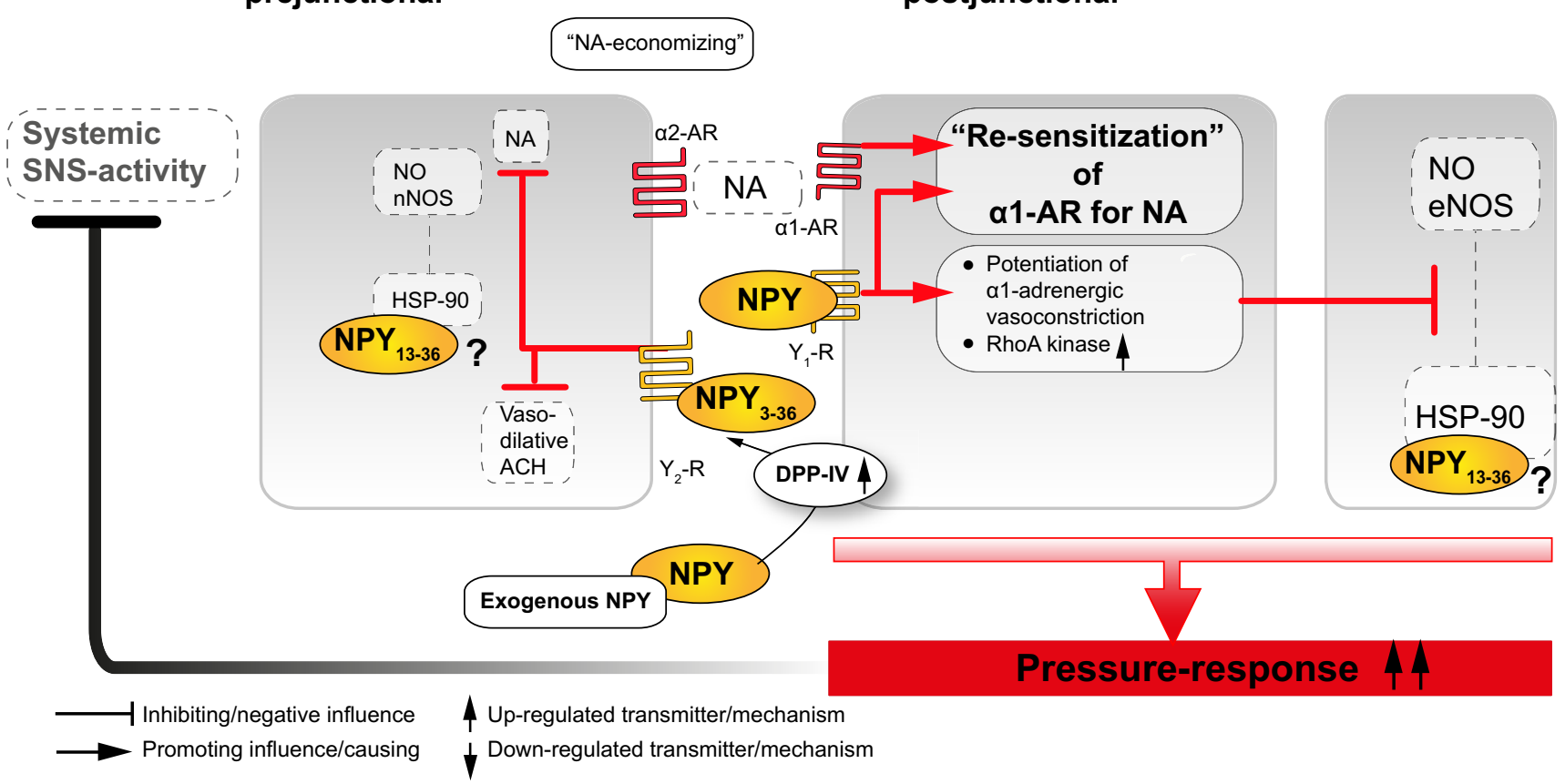

Fig. 4. Hypothesis on dysregulated sympathetic nervous system in portal hypertensive mesenteric arteries. (A) Regarding the mesenteric arterial smooth muscle, chronically increased NA levels lead to pre- and postjunctional effects resulting in decreased pressure response. (B) External NPY modulates vasocontrictive NA effects via postjunctional $\mathrm{Y}_{1}$ receptor-mediated and $\mathrm{Y}_{1 / 2 / 5}$ receptor-mediated prejunctional effects (e.g., economizing NA release). For a detailed explanation see the Discussion section. $\alpha_{1 / 2}$-AR, $\alpha_{1 / 2}$-adrenoceptor; ACH, acethylcholine; DPP-IV, dipeptidylpeptidase-IV; ET-1/3, endothelin-1/3; HSP-90, heat shock protein-90; NO, nitric oxide; eNOS, endothelial nitric oxide synthase; nNOS, neuronal nitric oxide synthase; SNS, sympathetic nervous system. (This figure appears in color on the web.)

endothelin-1 and -3 are elevated [34,35] and therefore could contribute to selective inhibition of NPY release. Finally, the observed accelerated decline of PNS-induced NA release and dysregulated NA release in cirrhosis may reflect reduced expression of genes regulating axonal transport, vesicle trafficking, and exocytosis of catecholamines [11]. Therefore, sympathetic nerve atrophy could result in a compensatory increased initial release but faster wastage of NA from presynaptic vesicles. However, the observed local splanchnic deficit in PNS-induced NPY release is in apparent contrast to increased systemic NPY levels reported in patients with cirrhosis [36]. Nonetheless, systemic concentrations only reflect the "spill-over" of neurotransmitters from the synaptic 
cleft in multiple compartments into the circulation, representing remaining NPY after degradation/metabolism at various sites [4]. Here portal hypertensive rats not only presented well-known vascular hyporeactivity $[1-4,15,16]$, but an accelerated decrease in pressure response to repetitive PNS. This desensitization process seems to be physiological, being present also in healthy rats, but the extent is exaggerated in portal hypertension. Regarding this, the hypothesis of pronounced postjunctional "smooth muscle desensitization" has to be supplemented, for it seems that the pronounced depletion of pressure response to sustained SNS activity is at least partly attributable to an enhanced prejunctional depletion of NA release and deficient NPY release. This concurs with the fact that vascular hyporesponsiveness in cirrhosis is not due to changes in the affinity and/or number of postsynaptic $\alpha_{1}-1$ adrenoceptors [37]. Since NPY is a potent amplifier of the vasoconstrictive response to NA [13-16], it appears that not the absolute amount, but rather the proportion of transmitter/cotransmitter release is essential for vasoconstriction. This was underscored by altered NA/PR and NA/NPY ratios in cirrhosis. In conjunction with the observed NA depletion but more robust stable splanchnic NPY release, it is tempting to speculate that NPY represents a basal nerval reserve function enabling compensation for loss of NA release in situations of extensive SNS activation, e.g., gastrointestinal hemorrhage. In portal hypertensive mesenteric vasculature, however, this reserve function appears vastly limited.

Approximately $30 \%$ of vasoconstriction produced by the SNS in mesenteric vasculature depends on NPY [20]. This substantiates the clinical significance of the observed lack of splanchnic NPY release. Furthermore, NPY deficiency could explain our previous findings, namely why the following effects of external NPY were more markedly pronounced in portal hypertension: NPY (i) inhibited eNOS- and nNOS-mediated NO production and its vasodilating effect $[16,38]$, (ii) enhanced vasoconstrictive Rho-A kinase expression and activity [38], and (iii) reduced portal pressure and portal blood flow [38]. Here exogenous NPY not only corrected mesenteric PNS-induced pressure response in portal hypertension, but markedly slowed the desensitization process, restoring vascular sensitivity to PNS-induced endogenous NA. NPY-induced potentiation of $\alpha_{1}$-adrenergic vasoconstriction via postsynaptic $Y_{1}$ receptors is well known $[13,14,38]$. Besides, prejunctional NPY effects have been studied in healthy rats. NPY exerts a negative feedback on NA release at the synaptic cleft, limiting further NA release by activation of a variety of receptors including the Y1, Y2, and Y5 receptor [39]. In this study, NPY suppressed NA release more potently in cirrhosis. The ratio of NA release to PNS-induced pressure response, indicating the NA amount required for vasoconstriction, was greatly diminished by exogenous NPY in cirrhosis. The pronounced post and prejunctional NPY action in portal hypertension also matches our reported results of nNOS and NO overproduction in mesenteric nerves and eNOS upregulation leading to arterial vasodilation in portal hypertension [40,41]. Kolo et al. [42] showed that the vasoconstrictive ability of NA in the rat mesenteric arterial bed was attenuated by NO, whereas the vasoconstrictive ability of NPY was unaffected. This fits with the enhanced NPY efficacy in cirrhosis despite NO overproduction, especially because in our experiments endothelium was intact. Previously, we showed that heat-shock protein-90 (HSP-90) acts as signaling mediator of nNOS- and eNOS dependent vasodilation in mesenteric arteries, and that increased nitrergic vasorelaxation observed in portal

\section{JOURNAL OF HEPATOLOGY}

hypertension is mediated largely by HSP-90 [43,44]. However, the reported strong binding of processed NPY N3-36 to HSP-90 might outline a mechanism of NPY-mediated NOS/NO regulation [45]. Furthermore, the proteolytic processing of NPY leads to selective activation of presynaptic Y2 receptors via $\mathrm{NPY}_{3-36}$ [32]. Therefore, the observed Y2 receptor-associated decrease of NA release might be more pronounced in cirrhosis because of the above mentioned elevated DPP-IV-activity [30] and thus increased proteolytic processing of NPY with subsequent selective presynaptic Y2 receptor stimulation. Moreover, cholinergic M3 receptor-induced vasodilation in the perfused rat mesentery is well known [46]. Interestingly, NPY mediates inhibition of sympathetic cholinergic vasodilatation via Y2 receptors but not of sympathetic adrenergic vasoconstriction [47], giving another explanation of how specific Y2 receptor binding might inhibit vasodilation, particularly in cirrhosis.

In summary, we report that mesenteric vasculature of cirrhotic rats presents with dysbalanced mesenteric sympathetic neurotransmitter release, namely increased NA release but diminished NPY release, resulting in vasoconstrictive hyporeactivity (Fig. 4A). Exogenous NPY has little effect on pressure response in controls but markedly augments PNS-induced pressure response, reduces PNS-induced vascular desensitization and suppresses NA release in cirrhosis, thereby probably economizing NA release. These effects evidence pronounced pre- and postjunctional NPY effects in portal hypertensive splanchnic arteries (Fig. 4B).

In humans, splanchnic vasoconstriction during exercise is associated with local NPY and NA release, and additional elevation in plasma NPY and catecholamines after propranolol administration is probably due to increased SNS activity and/or decreased disposal [48]. This reveals that NPY also might play an important role in the splanchnic mechanism of action of non-selective $\beta$-blockers in cirrhosis. NPY infusion in healthy humans has been shown to: (i) markedly reduce splanchnic and slightly lower renal blood flow and (ii) cause interesting metabolic changes, including inhibition of splanchnic glycogenolysis and appetite regulation $[49,50]$. Therefore, NPY infusion should be tested in patients with portal hypertension for its potential impact on splanchnic hemodynamics with careful monitoring of renal function and metabolic markers.

\section{Financial support}

This study was supported by the Regensburger Forschungsförderung in der Medizin (ReForM, to LM).

\section{Conflict of interest}

The authors who have taken part in this study declared that they do not have anything to disclose regarding funding or conflict of interest with respect to this manuscript.

\section{Acknowledgements}

The authors gratefully acknowledge M. Munz, P. Dietrich sen., G. Hack, and L. Rauch for the excellent technical support.

\section{Supplementary data}

Supplementary data associated with this article can be found, in the online version, at http://dx.doi.org/10.1016/j.jhep.2012.09.027. 
Research Article

\section{References}

[1] Wiest R, Groszmann RJ. The paradox of nitric oxide in cirrhosis and porta hypertension: Too much, not enough. Hepatology 2002;35:478-491.

[2] Hennenberg M, Trebicka J, Biecker E, Schepke M, Sauerbruch T, Heller J. Vascular dysfunction in human and rat cirrhosis: Role of receptor-desensitizing and calciumsensitizing proteins. Hepatology 2007;45:495-506.

[3] Llach J, Ginès P, Arroyo V, Rimola A, Titó L, Badalamenti S, et al. Prognostic value of arterial pressure, endogenous vasoactive systems, and renal function in cirrhotic patients admitted to the hospital for the treatment of ascites. Gastroenterology 1988;94:482-487.

[4] Braillon A, Gaudin C, Poo JL, Moreau R, Debaene B, Lebrec D. Plasma catecholamine concentrations are a reliable index of sympathetic vascular tone in patients with cirrhosis. Hepatology 1992;15:58-62.

[5] Gaudin C, Ruget G, Braillon A, Selz F, Cuche JL, Lebrec D. Portal and arterial free and conjugated noradrenaline in two models of portal hypertension in rats. Life Sci 1989;45:1333-1339.

[6] Shaldon C, Peacock JH, Walker RM, Palmer DB, Badrick FE. The portal venous content of adrenaline and noradrenaline in portal hypertension. Lancet 1961;1:957-961.

[7] Henriksen JH, Ring-Larsen H, Christensen NJ. Hepatic intestinal uptake and release of catecholamines in alcoholic cirrhosis Evidence of enhanced hepatic intestinal sympathetic nervous activity. Gut 1987;28:1637-1642.

[8] MacGilchrist A, Howes LG, Hawksby C, Reid JL. Plasma noradrenaline in cirrhosis: a study of kinetics and temporal relationship to ascites formation. Eur J Clin Invest 1991;21:238-243.

[9] Nicholls KM, Shapiro MD, Van Putten VJ, Kluge R, Chung HM, Bichet DG, et al Elevated plasma norepinephrine concentrations in decompensated cirrhosis. Association with increased secretion rates, normal clearance rates, and suppressibility by central blood volume expansion. Circ Res 1985;56: 457-461.

[10] Coll M, Martell M, Raurell I, Ezkurdia N, Cuenca S, Hernández-Losa J, et al. Atrophy of mesenteric sympathetic innervation may contribute to splanchnic vasodilation in rat portal hypertension. Liver Int 2010;30:593-602.

[11] Coll M, Genescà J, Raurell I, Rodríguez-Vilarrupla A, Mejías M, Otero T, et al. Downregulation of genes related to the adrenergic system may contribute to splanchnic vasodilation in rat portal hypertension. J Hepatol 2008;49:43-51.

[12] Ezkurdia N, Coll M, Raurell I, Rodriguez S, Cuenca S, González A. Blockage of the afferent sensitive pathway prevents sympathetic atrophy and hemodynamic alterations in rat portal hypertension. Liver Int 2012;32:1295-1305.

[13] Wahlestedt C, Edvinsson L, Ekblad E, Hakanson R. Neuropeptide Y potentiates noradrenaline evoked vasoconstriction: mode of action. J Pharmaco Exp Ther 1985;234:735-741

[14] Cortés V, Donoso MV, Brown N, Fanjul R, López C, Fournier A, et al Synergism between neuropeptide $\mathrm{Y}$ and norepinephrine highlights sympathetic cotransmission: studies in rat arterial mesenteric bed with neuropeptide Y, analogs, and BIBP 3226. J Pharmacol Exp Ther 1999;289: 1313-1322.

[15] Wiest R, Jurzik L, Moleda L, Froh M, Schnabl B, von Hörsten S, et al. Enhanced Y1-receptor-mediated vasoconstrictive action of neuropeptide Y (NPY) in superior mesenteric arteries in portal hypertension. J Hepatol 2006;44: 512-519.

[16] Wiest R, Jurzik L, Herold T, Straub RH, Schölmerich J. Role of NPY for vasoregulation in the splanchnic circulation during portal hypertension. Peptides 2007;28:396-404.

[17] Tsujimoto G, Honda K, Hoffman BB, Hashimoto K. Desensitization of postjunctional alpha 1- and alpha 2-adrenergic receptor-mediated vasopressor responses in rat harboring pheochromocytoma. Circ Res 1987;61:86-98

[18] Rosenbaum JS, Zera P, Umans VA, Ginsburg R, Hoffman BB. Desensitization of aortic smooth-muscle contraction in rats harboring pheochromocytoma. J Pharmacol Exp Ther 1986;238:396-400.

[19] Donoso MV, Brown N, Carrasco C, Cortes V, Fournier A, Huidobro-Toro JP. Stimulation of the sympathetic perimesenteric arterial nerves releases neuropeptide $\mathrm{Y}$ potentiating the vasomotor activity of noradrenaline: involvement of neuropeptide Y-Y1 receptors. J Neurochem 1997;69: 1048-1059.

[20] Han S, Yang CL, Chen X, Naes L, Cox BF, Westfall T. Direct evidence for the role of neuropeptide $\mathrm{Y}$ in sympathetic nerve-stimulation-induced vasoconstriction. Am J Physiol 1998;274:H290-H294.

[21] McGregor DD. The effect of sympathetic nerve-stimulation on vasoconstrictor responses in perfused mesenteric vessels of the rat. J Physiol 1965;177:21-30.

[22] Sikuler E, Kravetz D, Groszmann RJ. Evolution of portal hypertension and mechanisms involved in its maintenance in a rat model. Am J Physiol 1985;248:G618-G625
[23] Capellino S, Cosentino M, Wolff C, Schmidt M, Grifka J, Straub RH Catecholamine-producing cells in the synovial tissue during arthritis: modulation of sympathetic neurotransmitters as new therapeutic target. Ann Rheum Dis 2010;69:1853-1860.

[24] Rosas-Ballina M, Olofsson PS, Ochani M, Valdés-Ferrer SI, Levine YA, Reardon $\mathrm{C}$, et al. Acetylcholine-synthesizing $\mathrm{T}$ cells relay neural signals in a vagus nerve circuit. Science 2011;334:98-101.

[25] Straub RH, Grum F, Strauch U, Capellino S, Bataille F, Bleich A, et al. Antiinflammatory role of sympathetic nerves in chronic intestinal inflammation. Gut 2008;57:911-921.

[26] Capellino S, Weber K, Gelder M, Härle P, Straub RH. First appearance and location of catecholaminergic cells during experimental arthritis and elimination by chemical sympathectomy. Arthritis Rheum 2012;64: 1110-1118.

[27] Haass M, Cheng B, Richardt G, Lang RE, Schömig A. Characterization and presynaptic modulation of stimulation-evoked exocytotic co-release of noradrenaline and neuropeptide $\mathrm{Y}$ in guinea pig heart. Naunyn Schmiedebergs Arch Pharmacol 1989;339:71-78.

[28] Pernow J, Lundberg JM. Modulation of noradrenaline and neuropeptide $Y$ (NPY) release in the pig kidney in vivo: involvement of alpha-2, NPY and angiotensin II receptors. Naunyn Schmiedebergs Arch Pharmacol 1989;340:379-385.

[29] Hall GT, Gardner TD, Potter EK. Attenuation of long-lasting effects of sympathetic stimulation after repeated stimulation. Circ Res 1990;67: 193-198.

[30] Lakatos PL, Firneisz G, Rákóczy G, Selmeci L, Szalay F. Elevated serum dipeptidyl peptidase IV (CD26, EC 3.4.14.5) activity in patients with primary biliary cirrhosis. J Hepatol 1999;30:740.

[31] Palmieri FE, Ward PE. Dipeptidyl(amino)peptidase IV and post proline cleaving enzyme in cultured endothelial and smooth-muscle cells. Adv Exp Med Biol 1989;247A:305-311.

[32] Mentlein R, Dahms P, Grandt D, Krüger R. Proteolytic processing of neuropeptide Y and peptide YY by dipeptidyl peptidase IV. Regul Pept 1993;49:133-144.

[33] Hoang D, Macarthur H, Gardner A, Westfall TC. Endothelin-induced modulation of neuropeptide $\mathrm{Y}$ and norepinephrine release from the rat mesenteric bed. Am J Physiol Heart Circ Physiol 2002;283:H1523-H1530.

[34] Asbert M, Ginès A, Ginès P, Jiménez W, Clària J, Saló J, et al. Circulating levels of endothelin in cirrhosis. Gastroenterology 1993;104:1485-1491.

[35] Bernardi M, Gulberg V, Colantoni A, Trevisani F, Gasbarrini A, Gerbes AL. Plasma endothelin-1 and -3 in cirrhosis: relationship with systemic hemodynamics, renal function and neurohumoral systems. J Hepatol 1996:24:161-168.

[36] Wiest R, Moleda L, Zietz B, Hellerbrand C, Schölmerich J, Straub R. Uncoupling of sympathetic nervous system and hypothalamic-pituitaryadrenal axis in cirrhosis. J Gastroenterol Hepatol 2008;23:1901-1908.

[37] Liao JF, Yu PC, Lin HC, Lee FY, Kuo JS, Yang MC. Study on the vascular reactivity and alpha 1- adrenoceptors of portal hypertensive rats. $\mathrm{Br} \mathrm{J}$ Pharmacol 1994;111:439-444.

[38] Moleda L, Trebicka J, Dietrich P, Gäbele E, Hellerbrand C, Straub RH, et al. Amelioration of portal hypertension and the hyperdynamic circulatory syndrome in cirrhotic rats by neuropeptide $\mathrm{Y}$ via pronounced splanchnic vasoaction. Gut 2011;60:1122-1132.

[39] Westfall TC, Naes L, Gardner A, Yang CL. Neuropeptide Y induced attenuation of catecholamine synthesis in the rat mesenteric arterial bed. J Cardiovasc Pharmacol 2006; 47:723-728.

[40] Jurzik L, Froh M, Straub RH, Schölmerich J, Wiest R. Up-regulation of nNOS and associated increase in nitrergic vasodilation in superior mesenteric arteries in pre-hepatic portal hypertension. J Hepatol 2005;43:258-265.

[41] Wiest R, Shah V, Sessa WC, Groszmann RJ. NO overproduction by eNOS precedes hyperdynamic splanchnic circulation in portal hypertensive rats. Am J Physiol 1999;276:G1043-G1051.

[42] Kolo LL, Westfall TC, Macarthur H. Nitric oxide decreases the biological activity of norepinephrine resulting in altered vascular tone in the rat mesenteric arterial bed. Am J Physiol Heart Circ Physiol 2004;286: H296-H303.

[43] Moleda L, Jurzik L, Froh M, Gäbele E, Hellerbrand C, Straub RH, et al. Role of HSP-90 for increased nNOS-mediated vasodilation in mesenteric arteries in portal hypertension. World J Gastroenterol 2010;16:1837-1844.

[44] Shah V, Wiest R, Garcia-Cardena G, Cadelina G, Groszmann RJ, Sessa WC. Hsp90 regulation of endothelial nitric oxide synthase contributes to vascular control in portal hypertension. Am J Physiol 1999;277:G463-G468.

[45] Ishiwatari-Hayasaka H, Maruya M, Sreedhar AS, Nemoto TK, Csermely P, Yahara I. Interaction of neuropeptide Y and Hsp90 through a novel peptide binding region. Biochemistry 2003;42:12972-12980. 


\section{JOURNAL OF HEPATOLOGY}

[46] Hendriks MG, Pfaffendorf M, Van Zwieten PA. Characterization of the muscarinic receptor subtype mediating vasodilation in the rat perfused mesenteric vascular bed preparation. J Auton Pharmacol 1992;12: 411-420.

[47] Mahns DA, Revington ML, Runcie MJ, McCloskey DI, Potter EK. Inhibition of sympathetic cholinergic vasodilatation by a selective NPY Y2 receptor agonist in the gracilis muscle of anaesthetised dogs. J Auton Nerv Syst 1998;68:14-20.
[48] Ahlborg G, Lundberg JM. Splanchnic release of neuropeptide Y during prolonged exercise with and without beta-adrenoceptor blockade in healthy man. Clin Physiol 1991;11:343-351.

[49] Ahlborg G, Weitzberg E, Sollevi A, Lundberg JM. Splanchnic and renal vasoconstrictor and metabolic responses to neuropeptide $\mathrm{Y}$ in resting and exercising man. Acta Physiol Scand 1992;145:139-149.

[50] Mercer RE, Chee MJ, Colmers WF. The role of NPY in hypothalamic mediated food intake. Front Neuroendocrinol 2011;32:398-415. 


\section{Supplementary Material}

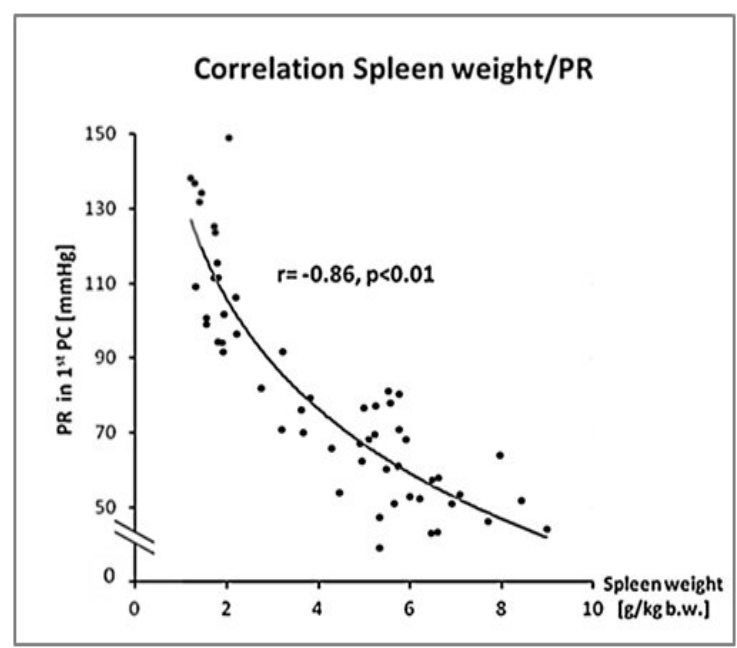

Suppl.-Fig. 1 Spleen weight and correlated PR to repetitive electric PNS [33Hz, 30 sec.] in $1^{\text {st }}$ PC. The correlation of relative spleen weight (as indicator for extension of portal hypertension) and mean PNS-induced PR is intense and significant and reveals a logarithmic expression $\left(\mathrm{R}^{2}=0.8\right)$. b.w., bodyweight. PC, perfusion-cycle. PNS, periarterial-nerve-stimulation. PR, pressure-response.

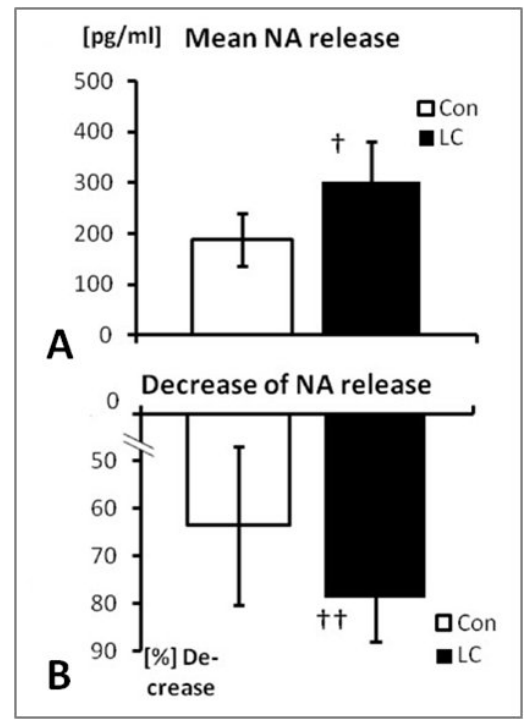

Suppl.-Fig. 2 PNS-induced NA-release in $1^{\text {st }}$ PC. Mean PNS-induced NA-release $(A)$ and percentage decrease of NA-release along the $1^{\text {st }}$ perfusion-cycle $(B)$ were significantly higher in LC rats than in control rats. $\dagger: \mathrm{p}<0.01$ vs. Con; $\dagger \dagger: \mathrm{p}<0.001$ vs. Con. LC, liver cirrhosis. NA, noradrenaline. PC, perfusion-cycle. PNS, periarterial-nerve-stimulation. 
Suppl.-Fig. 3 PR in dependency on PNS-induced NA-release in $1^{\text {st }}$

PC. Positive correlation and a marked vascular hyporeactivity to endogenous vasoconstrictors such as NA can be seen in portal hypertensive animals. However, the slope of PR in dependency on amount of NA-release was flattened in cirrhotic as compared to control rats. This demonstrates the known vascular hyporeactivity to vasoconstrictors of the mesenteric vasculature in portal hypertension. LC, liver cirrhosis. NA, noradrenaline. PC, perfusion-cycle. PNS, periarterial-nerve-stimulation. PR, pressure-response.

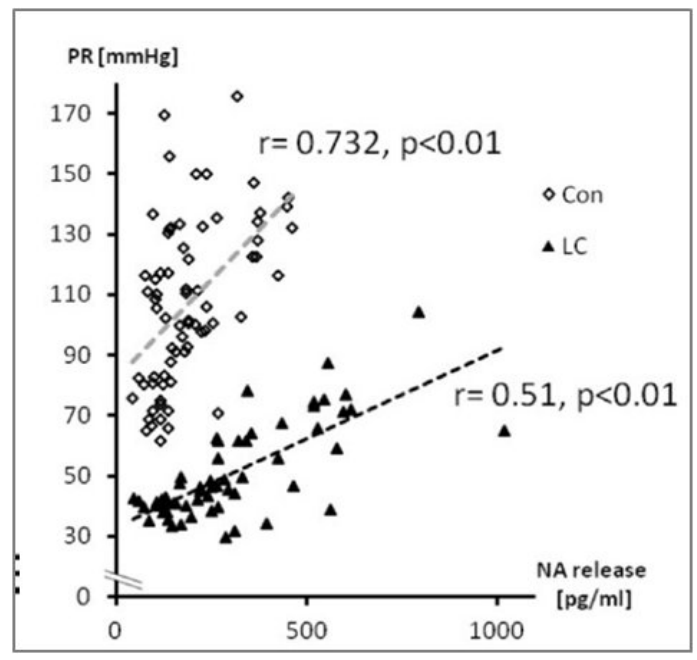

Suppl.-Fig. 4: NA-release in proportion to PR and NPY-release. (A): In order to estimate the efficacy of NA in causing vasoconstriction (PR), we calculated the ratio NA-release/PR. In fact, cirrhotic rats did exhibit higher NA/PR-ratios. The mean NA/PR-ratio in $1^{\text {st }} \mathrm{PC}$ was more than three-fold increased in cirrhotic rats. Unlike controls that showed constant NA/PRratios, NA/PR decreased in LC rats with cumulative PNS, pointing to the fact that the more pronounced decrease of NA-release may ameliorate vascular hyporeactivity to vasoconstrictors. $(B)$ : The NPY/NA-ratio was lower in LC rats, also underlining a (relative) deficiency in PNS-induced NPY-release in relation to augmented NA-release in mesenteric arteries in portal hypertensive conditions. LC, liver cirrhosis. NA, noradrenaline. PC, perfusion-cycle. PNS, periarterial-nerve-stimulation. PR, pressure-response.

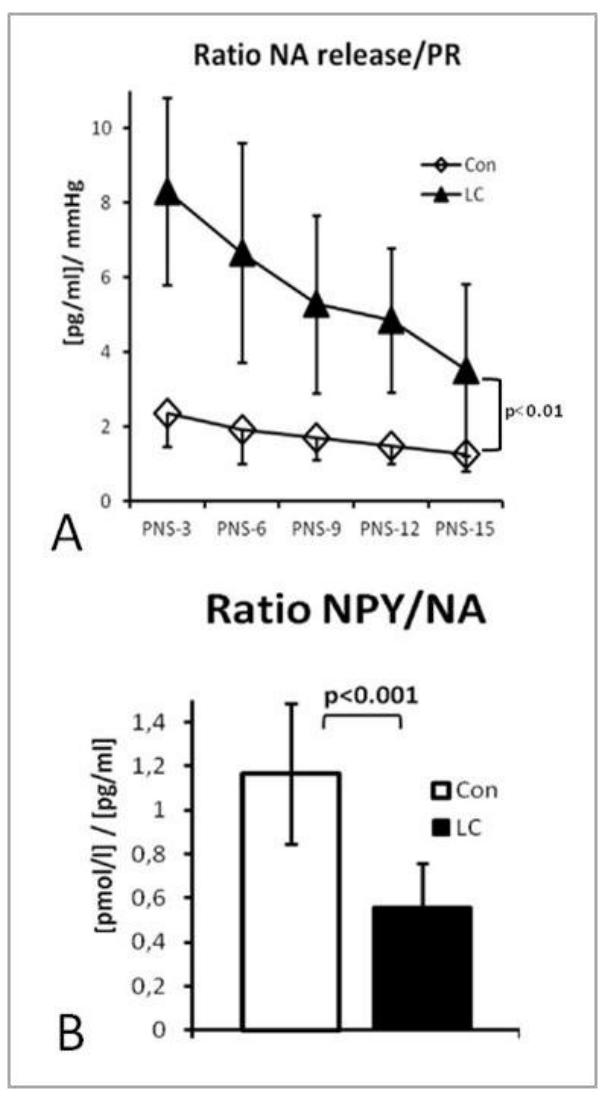




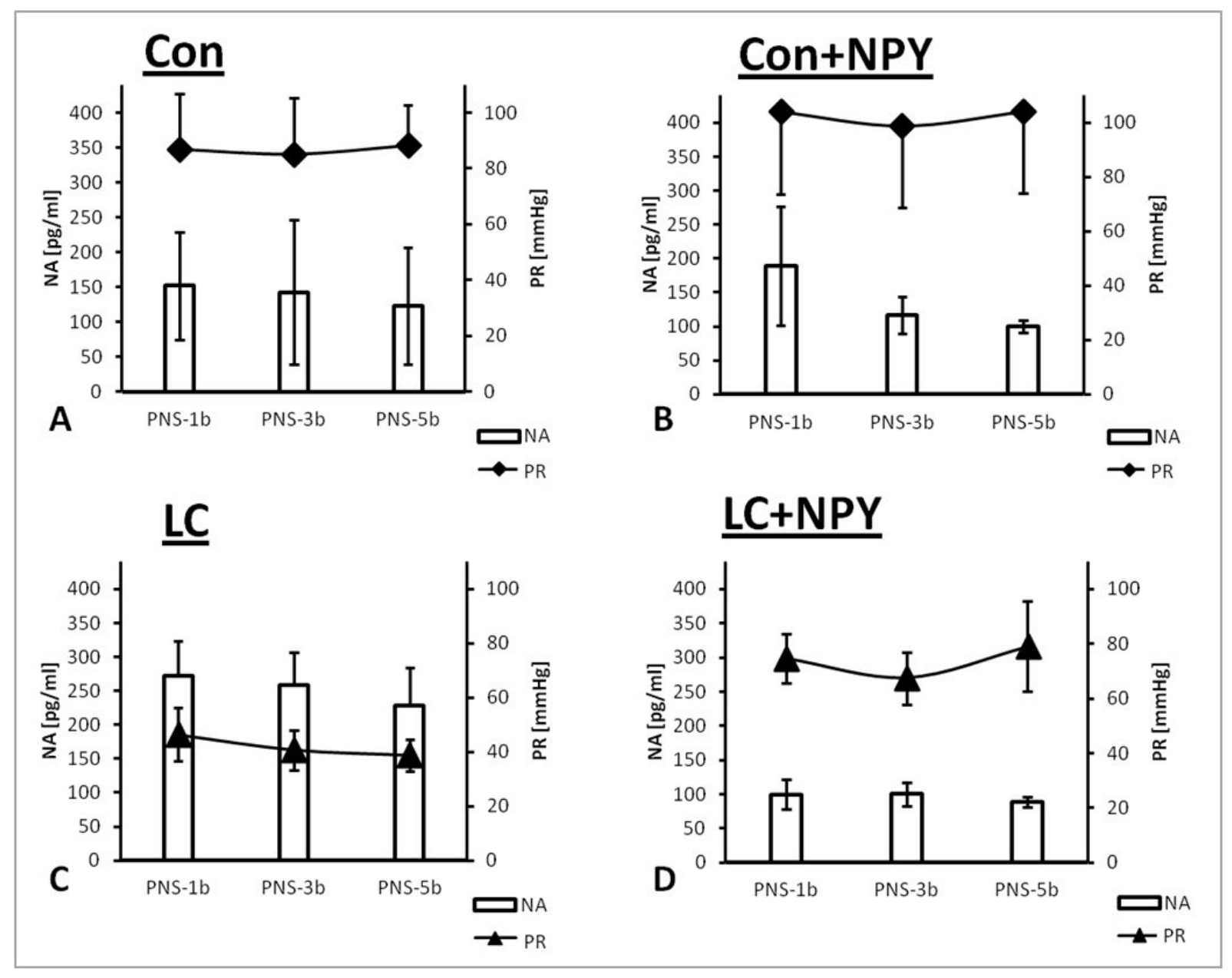

Suppl.-Fig. 5 Impact of exogenous NPY [50nM] on PR and NA-release in $2^{\text {nd }}$ PC. In portal hypertensive conditions, the impact of exogenous NPY is to elevate PNS-induced PR and to depress NA levels in LC rats significantly $(C, D)$, whereas these values are only tendentially modified in control rats $(A, B)$. LC, liver cirrhosis. NA, noradrenaline. PC, perfusion-cycle. PNS, periarterial-nerve-stimulation. PR, pressure-response. 


\section{Danksagung}

Ich möchte mich hiermit bei meinem Doktorvater, Herrn Prof. Dr. Reiner Wiest, Oberarzt der Universitätsklinik für Viszerale Chirurgie und Medizin am Inselspital Bern, für die Möglichkeit bedanken, diese Arbeit unter seiner Leitung durchzuführen. Insbesondere möchte ich mich für die hervorragende Betreuung, die ständige Diskussionsund Hilfsbereitschaft und das Teaching im Rahmen von Ergebnisbesprechungen sowie die stets lehrreichen gemeinsamen Ausarbeitungen von Vorträgen und wissenschaftlichen Kongressbeiträgen bedanken.

Mein weiterer Dank gilt Herrn Dr. Lukas Moleda von der Universitätsklinik Regensburg, der die Arbeit betreute und mir geduldig die notwendigen Techniken und Fertigkeiten im Labor beibrachte.

Meinem Vater Herrn Peter Dietrich danke ich für die Hilfe bei der Konstruktion der Vorrichtung zur elektrischen Nervenstimulation, die wesentlich zum Gelingen der Experimente beigetragen hat.

Herrn Manuel Munz von der Universität Bamberg danke ich für die kompetente Unterstützung bei der statistischen Auswertung der Ergebnisse.

Herrn Prof. Dr. Rainer Straub von der Universitätsklinik Regensburg möchte ich ganz herzlich für seine Erklärungen und Anregungen im Rahmen der Diskussion der Ergebnisse danken.

Besonderer Dank gilt auch Herrn Prof. Dr. Frieder Kees von der Universität Regensburg für die Hilfe bei der Messung der Katecholamine.

Frau Luise Rauch von der Universitätsklinik Regensburg danke ich für die Unterstützung bei der Messung des Neuropeptid Y.

Frau Gabi Hack von der Universitätsklinik Regensburg danke ich für die Hilfe bei der Arbeit mit den Versuchstieren.

Zuletzt möchte ich mich bei meiner Frau Susanne und meinen Eltern und Geschwistern für die uneingeschränkte, liebevolle und vielseitige Unterstützung bedanken. 